\title{
Consuming fructose-sweetened, not glucose- sweetened, beverages increases visceral adiposity and lipids and decreases insulin sensitivity in overweight/obese humans
}

\author{
Kimber L. Stanhope,1,2 Jean Marc Schwarz,3,4 Nancy L. Keim, ${ }^{5}$ Steven C. Griffen, 6 \\ Andrew A. Bremer,7 James L. Graham, ${ }^{1,2}$ Bonnie Hatcher, ${ }^{2}$ Chad L. Cox,, ${ }^{2}$ Artem Dyachenko, ${ }^{3}$ \\ Wei Zhang, ${ }^{6}$ John P. McGahan, ${ }^{8}$ Anthony Seibert, ${ }^{8}$ Ronald M. Krauss, ${ }^{9}$ Sally Chiu, ${ }^{9}$ \\ Ernst J. Schaefer, ${ }^{10}$ Masumi Ai, ${ }^{10}$ Seiko Otokozawa, ${ }^{10}$ Katsuyuki Nakajima, ${ }^{10,11}$ Takamitsu Nakano, ${ }^{11}$ \\ Carine Beysen, ${ }^{12}$ Marc K. Hellerstein,12,13 Lars Berglund,6,14 and Peter J. Havel1,2

\begin{abstract}
1Department of Molecular Biosciences, School of Veterinary Medicine, and 2Department of Nutrition, UCD, Davis, California, USA. ${ }^{3}$ College of Osteopathic Medicine, Touro University, Vallejo, California, USA. ${ }^{4}$ UCSF, San Francisco, California, USA. ${ }^{5}$ United States Department of Agriculture, Western Human Nutrition Research Center, Davis, California, USA. ${ }^{6}$ Department of Internal Medicine and ${ }^{7}$ Department of Pediatrics, School of Medicine, UCD, Sacramento, California, USA. ${ }^{8}$ Department of Radiology, UCD Medical Center, Sacramento, California, USA. ${ }^{9}$ Children's Hospital Oakland Research Institute, Oakland, California, USA. ${ }^{10}$ Lipid Metabolism Laboratory, Jean Mayer United States Department of Agriculture Human Nutrition Research Center on Aging at Tufts University, and Tufts University School of Medicine, Boston, Massachusetts, USA. ${ }^{11 D i a g n o s t i c ~ D i v i s i o n, ~ O t s u k a ~ P h a r m a c e u t i c a l ~ C o ., ~ T o k y o, ~ J a p a n . ~}$ ${ }^{12}$ KineMed, Emeryville, California, USA. ${ }^{13}$ Nutritional Sciences and Toxicology, University of California, Berkeley, California, USA. ${ }^{14}$ Veterans Affairs Northern California Health Care System, Sacramento, California, USA.
\end{abstract}

\begin{abstract}
Studies in animals have documented that, compared with glucose, dietary fructose induces dyslipidemia and insulin resistance. To assess the relative effects of these dietary sugars during sustained consumption in humans, overweight and obese subjects consumed glucose- or fructose-sweetened beverages providing $25 \%$ of energy requirements for 10 weeks. Although both groups exhibited similar weight gain during the intervention, visceral adipose volume was significantly increased only in subjects consuming fructose. Fasting plasma triglyceride concentrations increased by approximately $10 \%$ during 10 weeks of glucose consumption but not after fructose consumption. In contrast, hepatic de novo lipogenesis (DNL) and the 23-hour postprandial triglyceride AUC were increased specifically during fructose consumption. Similarly, markers of altered lipid metabolism and lipoprotein remodeling, including fasting apoB, LDL, small dense LDL, oxidized LDL, and postprandial concentrations of remnant-like particle-triglyceride and-cholesterol significantly increased during fructose but not glucose consumption. In addition, fasting plasma glucose and insulin levels increased and insulin sensitivity decreased in subjects consuming fructose but not in those consuming glucose. These data suggest that dietary fructose specifically increases DNL, promotes dyslipidemia, decreases insulin sensitivity, and increases visceral adiposity in overweight/obese adults.
\end{abstract}

\footnotetext{
Authorship note: Kimber L. Stanhope and Jean Marc Schwarz are co-first authors. Conflict of interest: M. Ai was supported by a research fellowship from Denka Seiken Co. L. Berglund is a shareholder of Pfizer. C. Beysen owns stock in and is an employee of KineMed. S.C. Griffen receives income from Bristol-Myers Squibb. P.J. Havel has a research contract with Novo Nordisk to test a proprietary compound for efficacy in delaying or preventing onset of diabetes in what we believe is a novel rat model of type 2 diabetes. M.K. Hellerstein owns stock in and has received consulting income from KineMed. R.M. Krauss has received consulting income from Merck, Merck-Schering Plough, and Isis Pharmaceuticals and has received grant support from Merck, Merck-Schering Plough, Sanofi-Aventis, and Metabolex. K. Nakajima has received consulting income from Denka Seiken Co. and Otsuka Pharmaceutical Co. S. Otokozawa was supported by a research fellowship from Kyowa Medex Co. J.A. Seibert is a consulting physicist for the American College of Radiology Imaging Network, National Lung Screening Trial.

Nonstandard abbreviations used: CCRC, Clinical and Translational Science Center's Clinical Research Center; DNL, de novo lipogenesis; GLM, general linear model; HFCS, high-fructose corn syrup; LPL, lipoprotein lipase; MSRF, metabolic syndrome risk factors; OGTT, oral glucose tolerance test; PROC MIXED, mixed procedures; RLP, remnant-like particle lipoprotein; RLP-C, RLP-cholesterol; RM, repeated measures; SAT, subcutaneous adipose tissue; sdLDL, small dense LDL; TG, triglyceride; VAT, visceral adipose tissue.

Citation for this article: J. Clin. Invest. 119:1322-1334 (2009). doi:10.1172/JCI37385
}

\section{Introduction}

Studies investigating the effects of fructose consumption in humans and animals have been comprehensively reviewed (1-4), and while strong evidence exists that consumption of diets high in fructose results in increased de novo lipogenesis (DNL), dyslipidemia, insulin resistance, and obesity in animals, direct experimental evidence that consumption of fructose promotes DNL, dyslipidemia, insulin resistance, glucose intolerance, and obesity in humans is lacking. Thus, we have investigated and compared the biological effects of the 2 major simple sugars in the diet, glucose and fructose, on BW and regional fat deposition and on indices of lipid and carbohydrate metabolism in older, overweight and obese men and women.

We sought to answer the following questions: (a) Does consumption of fructose with an ad libitum diet promote greater BW gain and have differential effects on regional adipose deposition and adipose gene expression compared with consumption of glucose with an ad libitum diet? (b) Does consumption of fructose induce dyslipidemia compared with consumption of glucose? (c) Is fructose-induced hypertriglyceridemia the result of increased rates 
of hepatic DNL and/or decreased triglyceride (TG) clearance? (d) Does consumption of fructose decrease glucose tolerance and insulin sensitivity? (e) Are there differences between the responses of older men and postmenopausal women to dietary fructose?

Consumption of fructose-sweetened but not glucose-sweetened beverages for 10 weeks increased DNL, promoted dyslipidemia, decreased insulin sensitivity, and increased visceral adiposity in overweight/obese adults.

\section{Results}

During the baseline phase of the study, subjects resided in the UCD Clinical and Translational Science Center's Clinical Research Center (CCRC) for 2 weeks and consumed an energy-balanced, high-complex carbohydrate (55\%) diet (Supplemental Table 1; supplemental material available online with this article; doi:10.1172/ JCI37385DS1). Procedures conducted during the baseline CCRC visit included a 24-hour serial blood collection, a 26-hour stable isotope infusion for determination of fractional DNL, fasting and postprandial postheparin blood sampling, an oral glucose tolerance test (OGTT) and disposal test, a gluteal adipose biopsy, and a CT scan of the abdomen. Subjects then began an 8-week outpatient intervention and consumed either fructose- $(n=17)$ or glucose-sweetened $(n=15)$ beverages at $25 \%$ of energy requirements with self-selected ad libitum diets. The subjects returned to the CCRC after 2 outpatient weeks for 2 days and then again for the final 2 weeks of the intervention for inpatient metabolic studies, during which the glucose- or fructose-sweetened beverages were consumed as part of an energy-balanced diet. Blood was collected over four 24-hour periods, during baseline and after 2, 8, and 10 weeks of intervention. The study design is outlined in Table 1.

Baseline characteristics and parameters. There were no significant differences between the 2 experimental groups in baseline anthropomorphic characteristics or in any of the measured metabolic parameters (Table 2).

Outpatient food intake, $B W$ and composition, adipose tissue gene expression, and blood pressure. During 24-hour food-intake recall interviews conducted on 6 outpatient days, both groups of subjects reported consuming significantly more energy than their calculated energy requirements. There were no significant differences between men and women or between subjects consuming glucose and subjects consuming fructose in fat, sugar, or alcohol intake as a percentage of energy intake or in the amount of energy consumed as a percentage of calculated energy requirements (Supplemental Table 2).

The changes in anthropomorphic outcomes are summarized in Table 3, and detailed analyses are presented in Supplemental Table 3. Despite comparable weight gain, there were differential effects of glucose and fructose on regional adipose deposition and gene expression. BW was stable during the 2 -week inpatient periods at both the beginning and end of the study. However, during the 8 -week outpatient intervention period, when the subjects consumed $25 \%$ of daily energy requirement as glucose- or fructosesweetened beverages along with ad libitum self-selected diets, both groups of subjects exhibited significant increases of BW (Figure 1A), fat mass, and waist circumference. Total and visceral adipose tissue (VAT) volumes were not significantly changed in subjects consuming glucose; however, subcutaneous adipose tissue (SAT) volume was significantly increased. In contrast, both total abdominal fat and VAT volume were significantly increased in subjects consuming fructose (Figure 1B).

SAT from the gluteal region was biopsied at 0 weeks and 10 weeks and analyzed for the expression of lipogenic and other genes (Supplemental Table 4). The percentage changes of gene expression at 10 weeks compared with baseline ( 0 weeks) were greater in subjects consuming glucose than in those consuming

Table 1

Twelve-week inpatient/outpatient, procedure, and diet schedule

\begin{tabular}{|c|c|c|c|c|c|c|c|}
\hline Study week & Monday & Tuesday & Wednesday & Thursday & Friday & Saturday & Sunday \\
\hline Week 2 Baseline & Inpatient ${ }^{A}$ & Inpatient ${ }^{A}$ & $\begin{array}{c}\text { Inpatient }^{A} \\
\text { DXA Postprandial } \\
\text { postheparin } \\
\text { blood draw }\end{array}$ & Inpatient ${ }^{A}$ & $\begin{array}{l}\text { InpatientA } \\
\text { OGTT Glucose } \\
\text { disposal test }\end{array}$ & Inpatient ${ }^{A}$ & Inpatient ${ }^{A}$ \\
\hline Week 1 Baseline & $\begin{array}{c}\text { Inpatient }{ }^{A} \\
\text { Gluteal adipose } \\
\text { biopsy }\end{array}$ & $\begin{array}{c}\text { InpatientB } \\
\text { 26-hour stable } \\
\text { isotope infusion }\end{array}$ & $\begin{array}{c}\text { Inpatient }{ }^{A} \\
\text { CT Scan }\end{array}$ & Inpatient ${ }^{A}$ & $\begin{array}{c}\text { Inpatient }{ }^{A} \\
\text { 24-hour } \\
\text { blood collection }\end{array}$ & $\begin{array}{l}\text { Inpatient }{ }^{A} \\
\text { Fasting postheparin } \\
\text { blood draw }\end{array}$ & Checkoutc \\
\hline Week 1-2 Intervention & Outpatientc & Outpatientc & Outpatientc & OutpatientC & Outpatientc & Outpatientc & Outpatient ${ }^{\mathrm{C}}$ \\
\hline Week 3 Intervention & Outpatientc & $\begin{array}{l}\text { InpatientD } \\
\text { Ad lib buffet }\end{array}$ & $\begin{array}{c}\text { InpatientE } \\
\text { 24-hour } \\
\text { blood collection }\end{array}$ & OutpatientB & OutpatientB & Outpatient ${ }^{\mathrm{B}}$ & Outpatient ${ }^{B}$ \\
\hline Week 4-8 Intervention & Outpatientc & OutpatientC & Outpatientc & Outpatientc & Outpatientc & Outpatientc & OutpatientC \\
\hline Week 9 Intervention & $\begin{array}{l}\text { InpatientD } \\
\text { Ad lib buffet }\end{array}$ & $\begin{array}{c}\text { Inpatient }{ }^{\mathrm{E}} \\
\text { 24-hour } \\
\text { blood collection }\end{array}$ & $\begin{array}{c}\text { InpatientE } \\
\text { DXA Postprandial } \\
\text { postheparin } \\
\text { blood draw }\end{array}$ & Inpatient ${ }^{E}$ & $\begin{array}{l}\text { InpatientE } \\
\text { Oral glucose } \\
\text { tolerance and } \\
\text { disposal test }\end{array}$ & InpatientE & InpatientE \\
\hline Week 10 Intervention & $\begin{array}{c}\text { InpatientE } \\
\text { Gluteal adipose } \\
\text { biopsy }\end{array}$ & $\begin{array}{c}\text { InpatientF } \\
\text { 26-hour stable } \\
\text { isotope infusion }\end{array}$ & $\begin{array}{l}\text { InpatientE } \\
\text { CT scan }\end{array}$ & InpatientE & $\begin{array}{c}\text { InpatientE } \\
\text { 24-hour } \\
\text { blood collection }\end{array}$ & $\begin{array}{l}\text { InpatientE } \\
\text { Fasting postheparin } \\
\text { blood draw }\end{array}$ & Checkout \\
\hline
\end{tabular}

\footnotetext{
${ }^{A}$ Energy-balanced diet: $55 \%$ of energy requirement complex carbohydrate; $30 \%$ fat; $15 \%$ protein. ${ }^{B}$ Steady-state energy-balanced diet: $55 \%$ of energy requirement complex carbohydrate; $30 \%$ fat; $15 \%$ protein. ${ }^{C} \mathrm{Ad}$ libitum usual diet plus $25 \%$ of energy requirement as sugar-sweetened beverage. ${ }^{\mathrm{D} A d}$ libitum food-intake trial plus $25 \%$ of energy requirement as sugar-sweetened beverage. EEnergy-balanced diet: $25 \%$ sugar-sweetened beverage; $30 \%$ complex carbohydrate; $30 \%$ fat; $15 \%$ protein. FSteady-state energy-balanced diet: $25 \%$ sugar beverage; $30 \%$ complex carbohydrate; $30 \%$ fat; $15 \%$ protein. DXA, dual energy x-ray absorptiometry.
} 
Table 2

Baseline anthropomorphic and metabolic parameters

\begin{tabular}{|c|c|c|c|c|}
\hline \multirow[b]{2}{*}{ Parameter } & \multicolumn{2}{|c|}{ Glucose } & \multicolumn{2}{|c|}{ Fructose } \\
\hline & $\begin{array}{c}\text { Male } \\
(n=7)\end{array}$ & $\begin{array}{c}\text { Female } \\
(n=8)\end{array}$ & $\begin{array}{c}\text { Male } \\
(n=9)\end{array}$ & $\begin{array}{c}\text { Female } \\
(n=8)\end{array}$ \\
\hline Age (yr) & $54 \pm 3$ & $56 \pm 2$ & $52 \pm 4$ & $53 \pm 2$ \\
\hline Weight (kg) & $88.4 \pm 2.9$ & $84.0 \pm 4.5$ & $89.3 \pm 2.9$ & $81.9 \pm 4.2$ \\
\hline BMI $\left(\mathrm{kg} / \mathrm{m}^{2}\right)$ & $29.3 \pm 1.1$ & $29.4 \pm 1.3$ & $28.4 \pm 0.7$ & $30.3 \pm 1.0$ \\
\hline Waist circumference (cm) & $98.9 \pm 2.6$ & $91.0 \pm 4.0$ & $97.3 \pm 3.3$ & $91.8 \pm 4.4$ \\
\hline Body fat $(\%)$ & $29.4 \pm 1.1$ & $43.2 \pm 1.5$ & $28.5 \pm 1.3$ & $39.6 \pm 2.2$ \\
\hline $\mathrm{TG}(\mathrm{mg} / \mathrm{dl})$ & $148 \pm 31$ & $145 \pm 23$ & $131 \pm 21$ & $159 \pm 30$ \\
\hline Total cholesterol (mg/dl) & $179 \pm 14$ & $193 \pm 10$ & $176 \pm 6$ & $198 \pm 15$ \\
\hline $\mathrm{HDL}(\mathrm{mg} / \mathrm{dl})$ & $36 \pm 3$ & $41 \pm 3$ & $39 \pm 4$ & $41 \pm 3$ \\
\hline LDL (mg/dl) & $124 \pm 5$ & $123 \pm 11$ & $107 \pm 7$ & $124 \pm 15$ \\
\hline Glucose (mg/dl) & $89 \pm 2$ & $89 \pm 3$ & $88 \pm 1$ & $90 \pm 1$ \\
\hline Insulin $(u \mathrm{U} / \mathrm{ml})$ & $14.3 \pm 3.2$ & $15.6 \pm 2.9$ & $16.3 \pm 2.5$ & $12.0 \pm 1.6$ \\
\hline
\end{tabular}

GLM 2-factor ANOVA (type of sugar and sexual phenotype). There were no significant differences among groups. Data represent mean \pm SEM. ing glucose but were markedly increased in subjects consuming fructose (Figure 2, A and B). Fasting (Figure 3, A and B) and postprandial apoB, the apoB/apoA1 ratio, and total and LDL cholesterol were also unchanged during consumption of glucose and increased during consumption of fructose. In both groups of subjects, plasma HDL concentrations were unchanged at 10 weeks but increased at the 2and 8-week time points.

In subjects consuming glucose, fasting small dense LDL (sdLDL) concentrations (Figure 3, C and D) initially decreased at 2 weeks and were not different from baseline at 10 weeks. In contrast, fasting sdLDL concentrations increased progressively in subjects consuming fructose. sdLDL was the lipid parameter most affected by preexisting metabolic syndrome risk factors (MSRF), with increases during fructose consumption more than 2-fold greater in subjects with $3 \mathrm{MSRF}$

fructose for stearoyl-CoA desaturase, fatty acid desaturase 1, and fatty acid desaturase 2 .

All subjects had normal blood pressure measurements at baseline, and blood pressure values did not change during the consumption of either fructose or glucose over the course of the 10-week intervention period (Table 3; Supplemental Table 5).

Lipid and lipoprotein concentrations, fractional hepatic DNL, and lipoprotein lipase activity. Plasma concentrations of lipid and lipoprotein parameters measured at 0 weeks, 2 weeks, 8 weeks, and 10 weeks with detailed analyses are presented in Supplemental Table 6.

In general, plasma lipid and lipoprotein concentrations increased markedly during fructose consumption and were unchanged during glucose consumption (Table 4). In exception, fasting TG concentrations increased in subjects consuming glucose but were unchanged in subjects consuming fructose $(2,8$, and 10 weeks vs. 0 weeks: $+1.0 \% \pm 5.5 \%,+1.0 \% \pm 5.0 \%$ and $+3.9 \% \pm 5.5 \% ; P=0.92)$. There was marked variability in the fasting TG responses to fructose consumption both within groups and within the individual subject. The mean SD of the percentage changes at 2 weeks, 8 weeks, and 10 weeks compared with 0 weeks in each subject was $13.4 \% \pm 1.5 \%$. In contrast to fasting TG, indices of postprandial TG - 23-hour AUC, TG exposure, postprandial TG peak - did not increase in subjects consum- than in subjects with 0 to 2 MSRF (Supplemental Table 7). Fasting oxidized LDL concentrations did not change in subjects consuming glucose but increased in subjects consuming fructose.

Fasting plasma remnant-like particle lipoprotein-TG (RLP-TG) and RLP-cholesterol (RLP-C) concentrations were unaffected by consumption of glucose or fructose (data not shown). In subjects consuming glucose, postprandial concentrations of RLP-TG (Figure 3, E and F) were unchanged; however RLP-C concentrations were increased at 8 weeks. During consumption of fructose, postprandial concentrations of both RLP-TG and RLP-C were increased. FFA exposure over 24 hours was increased in subjects consuming glucose but unchanged in subjects consuming fructose.

Increased DNL contributed to the increases of postprandial TG during fructose consumption. Fractional hepatic DNL was unchanged during glucose consumption, both in the fasting $(8.8 \% \pm 1.8 \%$ vs. $9.5 \% \pm 1.8 \% ; P=0.47)$ and postprandial states $(13.4 \% \pm 2.8 \%$ vs. $14.2 \% \pm 1.7 \% ; P=0.31)$. Fasting DNL was unaffected during fructose consumption $(9.9 \% \pm 1.3 \%$ vs. $8.3 \% \pm 0.9 \%$; $P=0.25)$, but postprandial DNLwas significantlyincreased $(11.4 \% \pm 1.3 \%$ vs. $16.9 \% \pm 1.4 \% ; P=0.021$ ) (Table 4 ). The 16 -hour AUC for fractional DNL was not increased compared with baseline in subjects consuming glucose $(54 \% \pm 17 \%$ vs. $60 \% \pm 8 \% \times 16 \mathrm{~h} ; P=0.69)$

\section{Table 3}

Baseline values and percentage changes in body composition and blood pressure after consumption of glucose- or fructose-sweetened beverages for 10 weeks

\begin{tabular}{|c|c|c|c|c|}
\hline Outcome variable & $\begin{array}{l}\text { Glucose } \\
\text { (0 weeks) }\end{array}$ & $\begin{array}{c}\text { Glucose } \\
\text { (\% change } 10 \text { weeks) }\end{array}$ & $\begin{array}{l}\text { Fructose } \\
\text { (O weeks) }\end{array}$ & $\begin{array}{c}\text { Fructose } \\
\text { (\% change } 10 \text { weeks) }\end{array}$ \\
\hline Systolic BP (mmHg) & $122 \pm 2$ & $0 \pm 2$ & $120 \pm 2$ & $+1 \pm 1$ \\
\hline Diastolic BP (mmHg) & $77 \pm 1$ & $-2 \pm 1$ & $76 \pm 1$ & $-1 \pm 1$ \\
\hline $\mathrm{BW}(\mathrm{kg})$ & $85.9 \pm 2.7$ & $+1.8 \pm 0.5^{A}$ & $85.7 \pm 2.6$ & $+1.4 \pm 0.3^{\mathrm{B}}$ \\
\hline Total body fat $(\mathrm{kg})$ & $30.7 \pm 2.2$ & $+3.2 \pm 0.6^{B}$ & $28.9 \pm 2.2$ & $+2.8 \pm 1.0^{\mathrm{A}}$ \\
\hline Waist circumference $(\mathrm{cm})$ & $94.6 \pm 2.6$ & $+1.7 \pm 0.6^{c}$ & $94.7 \pm 2.7$ & $+1.9 \pm 0.4^{\mathrm{B}}$ \\
\hline Total abdominal fat (cc) & $765 \pm 57$ & $+4.8 \pm 2.1$ & $683 \pm 55$ & $+8.6 \pm 3.0^{C}$ \\
\hline Extraabdominal fat (cc) & $522 \pm 59$ & $+4.6 \pm 1.4^{c}$ & $476 \pm 43$ & $+7.3 \pm 4.0$ \\
\hline Intraabdominal fat (cc) & $243 \pm 21$ & $+3.2 \pm 4.4$ & $207 \pm 21$ & $+14.0 \pm 5.5^{A}$ \\
\hline
\end{tabular}

${ }^{A} P<0.01 ;{ }^{B} P<0.001 ;{ }^{C} P<0.05$, paired Student's $t$ test, 10 weeks vs. 0 weeks. Data represent mean \pm SEM. 

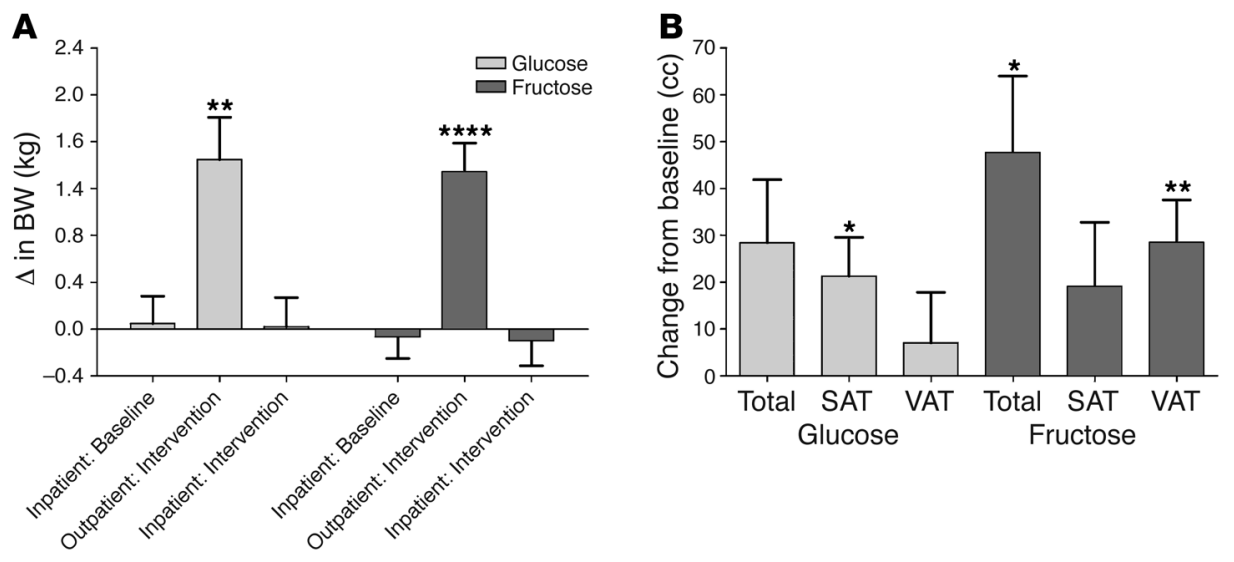

\section{Figure 1}

Changes of BW and abdominal fat. (A) Changes of BW during the 2-week inpatient baseline, 8-week outpatient intervention, and 2-week inpatient intervention periods. ${ }^{* \star} P<0.01 ;{ }^{* \star \star} P<0.0001$, day 56 outpatient:intervention vs. day 1 outpatient:intervention; paired Student's $t$ test. Glucose, $n=15$; fructose, $n=17$. (B) Changes of total abdominal adipose tissue, SAT, and VAT volume in subjects after consuming glucose- or fructose-sweetened beverages for 10 weeks. ${ }^{*} P<0.05$; ${ }^{* \star} P<0.01,10$ weeks vs. 0 weeks; paired Student's $t$ test. Glucose, $n=14$; fructose, $n=17$. Data represent mean \pm SEM.

but was significantly increased in subjects consuming fructose $(21 \% \pm 9 \%$ vs. $104 \% \pm 19 \% \times 16 \mathrm{~h} ; P=0.0043)$. The increase of the 16-hour AUC for fractional DNL during fructose consumption was significantly larger than that during glucose consumption $(83 \% \pm 22 \%$ vs. $7 \% \pm 14 \% \times 16$ h; $P=0.016)$ (Figure 4$)$.

Reduced TG clearance may also contribute to increases of postprandial TG in subjects consuming fructose. Postprandial postheparin lipoprotein lipase (LPL) activity tended to increase after 10 weeks of glucose consumption and to decrease after 10 weeks of fructose consumption, and the overall difference between the sugars was significant $(P=0.041)$. Fasting postheparin LPL activity was not significantly affected by consumption of either glucose or fructose (Table 4).

Plasma glucose, plasma insulin, and insulin sensitivity. Indices of insulin sensitivity/glucose tolerance at the measured time points with effects of sugar analyses are presented in Supplemental Table 8. In general, insulin sensitivity and glucose tolerance were not affected by the consumption of glucose but were decreased during the consumption of fructose (Table 5). Fasting glucose concentrations decreased in subjects consuming glucose but increased in subjects consuming fructose. Fasting insulin concentrations were unchanged during glucose consumption but were increased during consumption of fructose beverages. Glucose excursions, as assessed by the 3-hour AUC, increased in both groups of subjects during the OGTT (Figure 5, A and B). Insulin excursions were unchanged in subjects consuming glucose but increased in subjects consuming fructose (Figure 5, C and D). The insulin sensitivity index, assessed by the deuterated glucose disposal (5), was unchanged in subjects consuming glucose but decreased by $17 \%$ in subjects consuming fructose (Figure 5E). The magnitude of the changes of indices of insulin sensitivity during fructose consumption were not significantly affected by the number of MSRF (Supplemental Table 9).

Effect of sexual phenotype. The total and percentage increases of fat mass (men: $+4.4 \% \pm 0.8 \%$; women: $+1.5 \% \pm 0.7 \% ; P=0.020$ ) and intraabdominal fat volume (men: $+18.1 \% \pm 5.1 \%$; women: $-0.6 \% \pm 4.4 \%$; $P=0.049)$ were greater in men than in women. Men consuming fructose also had larger increases of intraabdominal fat compared with women consuming fructose $(P=0.033$; Supplemental Table $3)$. Fructose consumption resulted in larger increases of 24-hour
TG exposure, postprandial TG peak, and postprandial RLP-C in men compared with women (Supplemental Table 10). There were no significant differences in the effects of fructose on indices of glucose tolerance/insulin sensitivity between men and women (Supplemental Table 11). However, overall the changes in insulin sensitivity were different between men and women ( $P=0.033$; Supplemental Table 9), with women exhibiting significantly greater decreases of insulin sensitivity in response to sugar consumption than men. The insulin sensitivity index decreased by $10.2 \% \pm 12.1 \%$ in women consuming glucose but increased by $12.5 \% \pm 12.6 \%$ in men consuming glucose. The insulin sensitivity index decreased by $23.6 \% \pm 4.4 \%$ in women consuming fructose and by $11.7 \% \pm 5.6 \%$ in men consuming fructose.

Effects of energy intake during the previous day. Subjects consumed significantly more energy ad libitum on the days prior to the 2 -week and 8-week 24-hour serial blood collections than during the energy-balanced feeding that preceded the 0 -week and 10-week 24-hour serial blood collections (Supplemental Table 12). The previous day's energy intakes were included in the mixed procedures (PROC MIXED) repeated measures (RM) ANOVA model as a time-level covariable; therefore, the contribution and significance of the effect of the previous day's energy intake on the variation of the outcome response can be ascertained by the $F$ statistic and $P$ value of the covariable (Supplemental Table 13). Within subjects consuming fructose, postprandial apoB was the outcome most significantly affected by energy intake during the previous day; TG exposure, fasting apoB, and postprandial RLP-C were significantly affected as well.

\section{Discussion}

$B W$ and body fat. Given the comparable weight gain in the 2 groups of subjects, the differences in intraabdominal fat gain and in the gene expression of lipogenic enzymes from subcutaneous adipose biopsies suggest that fructose consumption may specifically promote lipid deposition in VAT, particularly in men, whereas glucose consumption appears to favor SAT deposition.

Dyslipidemia. In agreement with the results from this study, we and other investigators have reported that long-term consumption ( $\geq 2$ weeks) of fructose at $20 \%-25 \%$ of energy requirement did not 


\begin{tabular}{|c|c|c|c|c|}
\hline Outcome variable & $\begin{array}{l}\text { Glucose } \\
\text { (0 weeks) }\end{array}$ & $\begin{array}{c}\text { Glucose } \\
\text { (\% change } 10 \text { weeks) }\end{array}$ & $\begin{array}{l}\text { Fructose } \\
\text { (0 weeks) }\end{array}$ & $\begin{array}{c}\text { Fructose } \\
(\% \text { change } 10 \text { weeks })\end{array}$ \\
\hline Fasting TG (mg/dl) & $146 \pm 17$ & $+9.7 \pm 3.2^{\mathrm{A}}$ & $144 \pm 18$ & $+3.9 \pm 5.5$ \\
\hline 23-hour TG AUC $(\mathrm{mg} / \mathrm{dl} \times 23 \mathrm{~h})$ & $783 \pm 118$ & $-32.0 \pm 14.8$ & $808 \pm 167$ & $+99.2 \pm 31.5^{\mathrm{B}}$ \\
\hline Mean 24-hour TG (mg/dl) & $171 \pm 20$ & $+2.5 \pm 4.0$ & $163 \pm 21$ & $+18.2 \pm 5.8^{C}$ \\
\hline Postprandial TG peak (mg/dl) & $202.4 \pm 24.6$ & $+9.8 \pm 5.1$ & $211.1 \pm 28.3$ & $+38.1 \pm 7.9 c$ \\
\hline Fasting cholesterol (mg/dl) & $186 \pm 8$ & $+3.9 \pm 2.0$ & $186 \pm 8$ & $+10.1 \pm 1.3 \mathrm{D}$ \\
\hline Fasting LDL-C (mg/dl) & $123.4 \pm 5.9$ & $+3.6 \pm 3.0$ & $115.3 \pm 8.0$ & $+13.9 \pm 2.3^{\mathrm{E}}$ \\
\hline Fasting HDL-C (mg/dl) & $39 \pm 2$ & $-2.4 \pm 2.1^{F}$ & $40 \pm 3$ & $+3.5 \pm 1.8^{\mathrm{E}}$ \\
\hline Fasting apoB (mg/dl) & $86 \pm 6$ & $+3.0 \pm 3.6$ & $79 \pm 6$ & $+27.2 \pm 4.3^{B}$ \\
\hline Postprandial apoB (mg/dl) & $81 \pm 6$ & $+6.9 \pm 3.7$ & $74 \pm 6$ & $+25.0 \pm 4.9^{B}$ \\
\hline apoB/apoA1 (mg/dl) & $0.75 \pm 0.07$ & $+1.8 \pm 3.4$ & $0.63 \pm 0.06$ & $+22.4 \pm 4.3^{G}$ \\
\hline Fasting sdLDL-C (mg/dl) & $29.9 \pm 3.5$ & $+13.3 \pm 5.8 \mathrm{E}$ & $24.7 \pm 2.7$ & $+44.9 \pm 9.7^{G}$ \\
\hline Fasting oxLDL-C (U/I) & $53.3 \pm 3.0$ & $+0.7 \pm 3.0$ & $50.8 \pm 3.9$ & $+12.8 \pm 2.6 \mathrm{E}$ \\
\hline Postprandial RLP-TG (mg/dl) & $70.7 \pm 11.4$ & $+15.2 \pm 6.3$ & $82.6 \pm 16.5$ & $+78.6 \pm 19.8^{C}$ \\
\hline Postprandial RLP-C (mg/dl) & $10.1 \pm 1.4$ & $+3.7 \pm 5.4$ & $10.9 \pm 1.6$ & $+33.9 \pm 11.8 \mathrm{C}$ \\
\hline Mean 24-hour FFA (mEq/l) & $0.27 \pm 0.01$ & $+9.0 \pm 2.6^{F}$ & $0.28 \pm 0.02$ & $+0.9 \pm 3.8$ \\
\hline Fasting fractional DNL $(\%)$ & $8.8 \pm 1.8$ & $+12.3 \pm 10.3$ & $9.9 \pm 1.3$ & $-6.3 \pm 16.6$ \\
\hline Postprandial fractional DNL (\%) & $13.4 \pm 2.8$ & $+27.3 \pm 13.6$ & $11.4 \pm 1.4$ & $+75.4 \pm 25.6^{\mathrm{H}}$ \\
\hline Fasting postheparin LPL activity (U/I) & $79.0 \pm 5.8$ & $+0.2 \pm 4.4$ & $101.4 \pm 10.3$ & $+0.7 \pm 7.1$ \\
\hline Postprandial postheparin LPL activity (U/I) & $77.0 \pm 5.8$ & $+20.3 \pm 8.7$ & $106.9 \pm 11.1$ & $-5.4 \pm 8.9$ \\
\hline
\end{tabular}

$\mathrm{A} P<0.05 ; \mathrm{B} P<0.001 ;{ }^{C} P<0.0001$, PROC MIXED 3-factor (time, sexual phenotype, MSRF) RM ANOVA with previous day's energy intake as time-level covariable, effect of time. ${ }^{D} P<0.0001$; $E P<0.01$; $F P<0.05$; ${ }^{\mathrm{G}} P<0.001$, PROC MIXED 3-factor (time, sexual phenotype, MSRF) RM ANOVA, effect of time. ${ }^{\mathrm{H} P}<0.05$, paired Student's $t$ test, 10 weeks vs. 0 weeks. Data represent mean \pm SEM.

increase fasting TG concentrations in humans (6-12). However, increases of fasting TG concentrations have been reported after 2 or more weeks of fructose consumption at $15 \%-20 \%$ of energy requirements (13-18). The reason for these conflicting results is unclear but may be related to the marked within-group and within-individual variability we observed in fasting TG responses to dietary fructose.

Bantle et al. previously reported that a 6 -week diet providing $17 \%$ of energy from fructose increased postprandial TG concentrations compared with an isocaloric glucose diet in healthy men but not in healthy women (13). The increases of postprandial TG in men in the present study confirm those reported by Bantle et al.; however, the women consuming fructose from this study and our previous study (11) also had significantly increased TG AUCs compared with women consuming glucose. The women studied by Bantle et al. were leaner and younger than the women in the present study and of mixed menopausal status. Body fat (19), age (20), and menopausal status $(21,22)$ have all been shown to affect postprandial TG responses in women.

We have demonstrated that a mechanism by which fructose induces postprandial hypertriglyceridemia is through increased hepatic DNL. It has long been established that in contrast to the metabolism of glucose, fructose metabolism is independent of phosphofructose kinase regulation; thus, its uptake by the liver and its metabolism to DNL substrate is not limited by energy status (cytosolic ATP and citrate levels) (23). In addition, fructose may activate sterol receptor element-binding protein-1c independently of insulin, which activates genes involved in $\operatorname{DNL}(24,25)$. However, demonstrations that sustained fructose consumption increases DNL in humans are limited to an abstract (26) and an overfeeding study (800-1000 kcal/d fructose in excess of energy requirement) (27). This is the first study, to our knowledge, to demonstrate that prolonged fructose consump- tion but not glucose consumption increases hepatic fractional DNL in humans when measured during energy-balanced feeding.

The increased rate of fructose-induced DNL generates fatty acids for production of hepatic TG. Additionally, hepatic DNL limits fatty acid oxidation in the liver via production of malonyl-CoA, which reduces the entry of fatty acids into the mitochondria (28). Thus, fructose-induced DNL may increase hepatic lipid not only by supplying endogenous fatty acids but also by increasing the intrahepatic availability of fatty acids derived from the circulation (28). Increased hepatic lipid levels are associated with increased VLDL synthesis and secretion, specifically that of VLDL1 (29). apoB is essential for the intracellular assembly of TG into VLDL, and apoB degradation is reduced when hepatic lipid is increased (30). The positive correlations between the previous day's energy intake and postprandial apoB and TG concentrations in subjects consuming fructose suggest that positive energy balance also increases hepatic lipid availability.

While we propose that increased VLDL synthesis/secretion is the main contributor (31), the significantly different postprandial postheparin LPL responses between the 2 treatment groups suggest that reduced TG clearance might also contribute to fructose-induced postprandial hypertriglyceridemia. Both reduced postmeal exposure to insulin (32) and decreased insulin sensitivity (33) may have contributed to lowered postprandial LPL activity in subjects consuming fructose compared with those consuming glucose. It has been demonstrated that SAT is more sensitive to the effects of insulin in activating LPL than VAT (34); thus, the differential LPL responses may contribute to the increased fat deposition in SAT in subjects consuming glucose and increased fat deposition in VAT in subjects consuming fructose.

There is growing evidence linking increases of postprandial TG concentrations with proatherogenic conditions (35-40). This link 
A

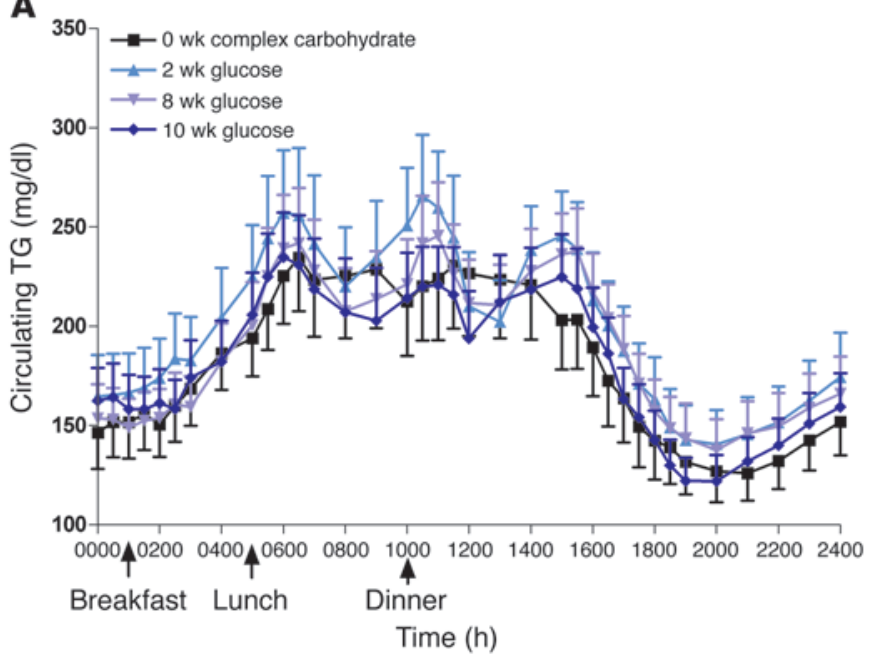

B

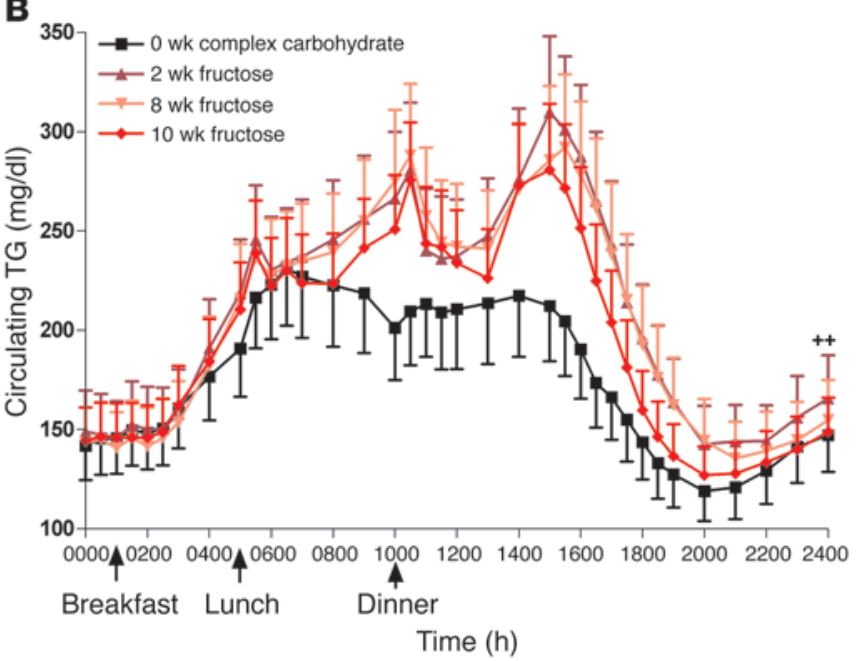

Figure 2

Plasma TG. 24-hour circulating TG concentrations in subjects before and after 2, 8, and 10 weeks of consuming glucose-sweetened beverages (A) or fructose-sweetened beverages (B). ${ }^{++} P<0.01$ PROC MIXED 3-factor RM ANOVA with prior day's energy intake covariable for 23-hour TG AUC. Glucose, $n=14$; fructose, $n=17$. Data represent mean \pm SEM.

may be due to lipoprotein remodeling induced by increased levels of VLDL1 and mediated by cholesteryl ester transfer protein (CETP) and hepatic lipase, which results in increased concentrations of sdLDL and RLP $(31,41-44)$. sdLDL is more easily oxidized than larger LDL particles (45), and accordingly, subjects consuming fructose also had significantly increased concentrations of oxidized LDL. The doubling of fructose-induced increases of both fasting and postprandial sdLDL concentrations in subjects with metabolic syndrome $(\mathrm{MSRF}=3)$ compared with subjects with 0-2 MSRF was striking. In all cases, the additional risk factor in the 5 subjects with MSRF 3 compared with the 5 subjects with MSRF 2 was the presence of fasting TG over $150 \mathrm{mg} / \mathrm{dl}$, suggesting that preexisting hypertriglyceridemia can exacerbate lipoprotein remodeling associated with fructose-induced increases of postprandial TG.

The unchanged FFA exposure in subjects consuming fructose is an important finding. It has been suggested that fructose consumption promotes development of the metabolic syndrome through increased adiposity and adipose insulin resistance, which leads to increased circulating and portal levels of FFA (46). The resulting increase in hepatic FFA uptake increases hepatic lipid availability and hepatic insulin resistance (47). However, the absence of an effect of fructose on systemic FFA suggests that fructose may promote insulin resistance by providing a more direct source of intrahepatic lipid via DNL (48).

Insulin sensitivity and glucose tolerance. We propose that the increased hepatic lipid resulting from fructose-induced DNL leads to hepatic insulin resistance $(49,50)$, possibly by increasing levels of diacylglycerol (49). Diacylglycerol is a known activator of novel PKC (51), and increases of both diacylglycerol and novel PKC activity are associated with lipid-induced insulin resistance $(52,53)$. It has been previously reported that consumption of 1,000 extra $\mathrm{kcal} / \mathrm{d}$ fructose along with ad libitum diet reduced insulin sensitivity in healthy subjects, whereas insulin sensitivity was unchanged in subjects consuming an extra $1,000 \mathrm{kcal} / \mathrm{d}$ glucose (54). Our results confirm this difference with a smaller quantity of fructose $(617 \pm 24 \mathrm{kcal} / \mathrm{d})$ consumed with an energy-balanced diet in a controlled metabolic setting for 3 days prior to testing. However, because the subjects gained BW and fat during the outpatient intervention, it is not known whether fructose consumption decreases insulin sensitivity to the same degree in the absence of BW and fat gain. Interestingly, the changes of BW or body fat and the change of insulin sensitivity were not correlated.

Recently, it was reported that the inclusion of fructose with an energy-balanced diet in men increased fasting glucose levels, but other indices of insulin sensitivity were unaffected (17). Factors that may contribute to the differences between these results and those of the present study include study duration, fructose exposure, subject weight gain, age, and baseline insulin sensitivity.

Effect of sexual phenotype. Bantle et al. (13) previously reported that fructose-induce postprandial TG responses were greater in men than in women; thus, the higher 24-hour TG exposure, postprandial TG peaks, and RLP-C concentrations we report in men compared with women were not unexpected. The finding that women had sdLDL increases comparable to those of men despite having lower TG responses to fructose consumption suggests that the TG threshold required to increase the production of sdLDL may be lower in women than men. The greater decrease of insulin sensitivity noted in women compared with men in response to sugar consumption was unexpected and contrasts with the sexual phenotype effect noted during a fructose overfeeding study (55). Indices of insulin sensitivity were decreased in healthy young men but were unchanged in healthy young women (55). Hepatic lipid accumulates when TG production exceeds FFA oxidation and VLDL production and secretion (56). It is possible that women in the current study exhibited larger decreases in insulin sensitivity than men due to decreased rates of VLDL production and secretion, which resulted in greater increases of hepatic lipid content. The younger women (55) may have accumulated less hepatic lipid than the older women due to their having increased rates of FFA oxidation (57). The overall effect of sexual phenotype on the changes of insulin sensitivity also contrasts with the sexual phenotype effect for the changes of intraabdominal fat, which increased more in men than in women. These opposing sexual phenotype effects also suggest that fructose decreases insulin sensitivity independently of visceral adiposity and FFA levels (48). 
A

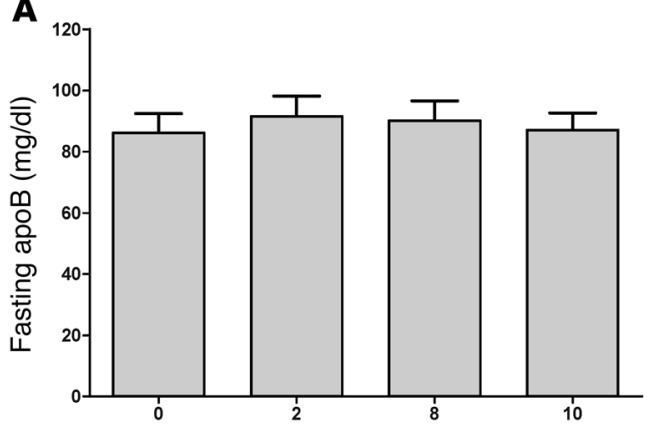

Glucose beverage intervention wk
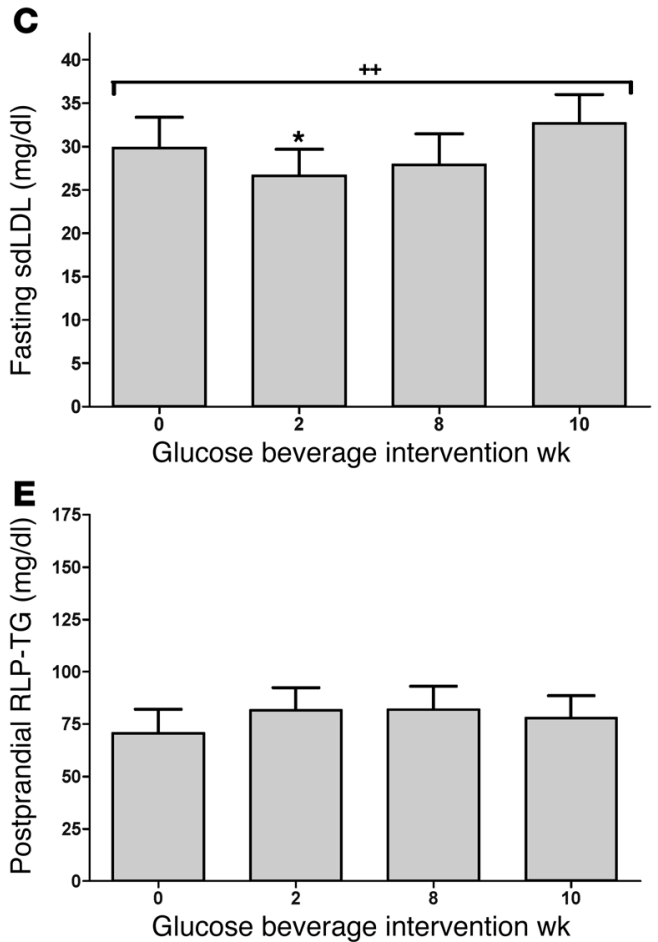

B

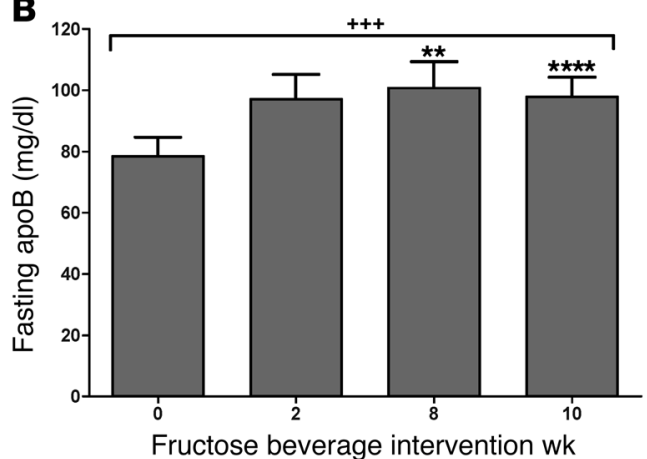

D
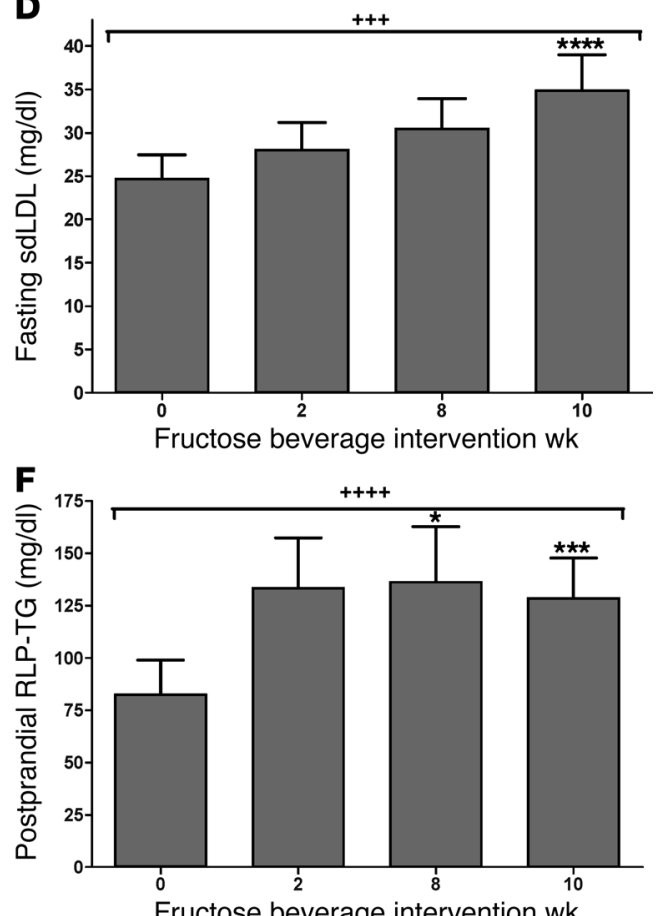

Figure 3

apoB, sdLDL, and RLP-TG. Fasting apoB concentrations in subjects before and after 2 , 8 , and 10 weeks of consuming glucose-sweetened beverages (A) or fructose-sweetened beverages (B). Fasting sdLDL concentrations in subjects before and after 2, 8, and 10 weeks of consuming glucosesweetened beverages (C) or fructose-sweetened beverages (D). Postprandial RLP-TG concentrations in subjects before and after 2, 8, and 10 weeks of consuming glucosesweetened beverages (E) or fructose-sweetened beverages $(\mathbf{F}) .{ }^{++} P<0.01 ;{ }^{+++} P<0.001$; ${ }^{++++} P<0.0001$, PROC MIXED 3-factor RM ANOVA (C and D) with prior day's energy intake covariable (A, B, E, and $\mathbf{F}) .{ }^{*} P<0.05 ;{ }^{*} P<0.01$; ${ }^{* * *} P<0.001 ;{ }^{* * * *} P<0.0001$, Tukey's multiple comparison test vs. 0 weeks. Glucose, $n=15$; fructose, $n=17$. Data represent mean \pm SEM.
Model. Figure 6 presents a proposed model for the divergent metabolic effects of glucose and fructose consumption.

Fructose and public bealth. While this study was designed to compare the biological effects of glucose and fructose consumption on lipid and carbohydrate metabolism, the potential implications of the results on public health is of interest. Foods and beverages in the US are typically sweetened with sucrose (50\% glucose and 50\% fructose) or high-fructose corn syrup (HFCS), which is usually $45 \%-58 \%$ glucose and $42 \%-55 \%$ fructose, rather than pure glucose or fructose. We have reported in a short-term study that the 23-hour postprandial TG profiles in male subjects consuming $25 \%$ energy as HFCS $(55 \%$ fructose) or sucrose were elevated to a degree similar to that observed when pure fructose-sweetened beverages were consumed (19). Therefore, it is uncertain whether the adverse effects of sucrose and HFCS consumption are "diluted" by their lower fructose content relative to pure fructose. Additional studies are needed to compare the longterm effects of consuming HFCS and/or sucrose with $100 \%$ fructose.

The amount of sugar consumed by the subjects in this study, $25 \%$ of energy requirements, is considerably higher than $15.8 \%$, the current estimate for the mean intake of added sugars by Americans (58). However, recent reports (59-63) suggest that the sugar intake from beverages alone approaches or exceeds $15 \%$ of energy in adolescents and adults up to 40 years of age. The large SDs in several of these reports suggest that at least $16 \%$ of the studied populations was consuming over $25 \%$ of daily energy requirements from sugar-sweetened beverages $(59,62,63)$.

Conclusions. We reached the following conclusions: (a) The increase in VAT in subjects consuming fructose and the increase in the expression of lipogenic genes in SAT in subjects consuming glucose suggest that fructose and glucose have differential effects on regional adipose distribution. We believe that these results are novel and warrant further investigation. (b) In addition to increases of postprandial TG and fasting and postprandial apoB, we show for what we believe is the first time that fructose consumption increases plasma concentrations of fasting sdLDL, oxidized LDL, and postprandial RLP-C and RLP-TG in older, overweight/obese men and women, whereas glucose consumption does not. These changes may be associated with increased risk of cardiovascular disease (30, 36, 45, 64-66). (c) Fructose consumption increased hepatic fractional DNL, and postprandial LPL activity was lower in subjects consuming fructose compared with those consuming glucose. These results suggest that both increased DNL and decreased LPL-mediated clearance contribute to 


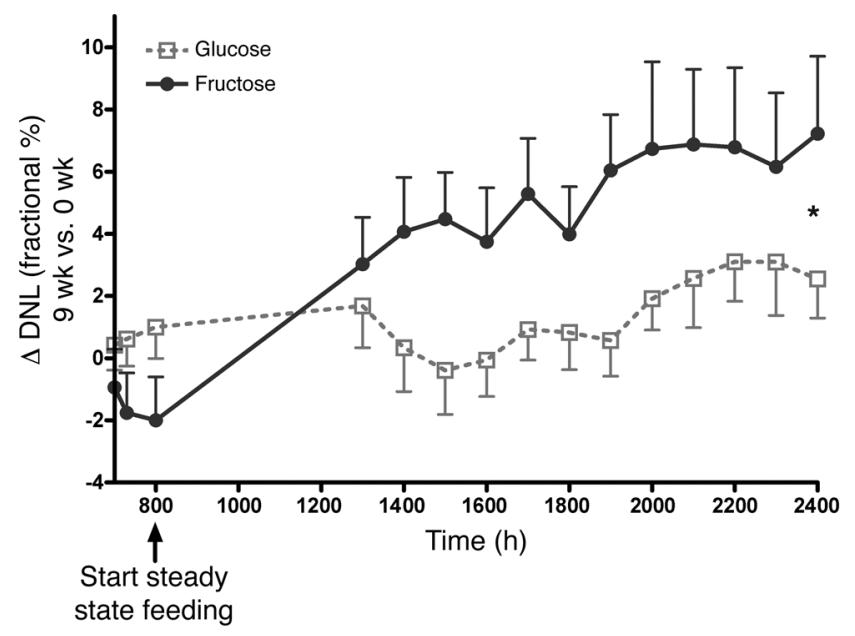

Figure 4

Hepatic fractional DNL. Change of fractional DNL before and during steady-state feeding of meals with glucose- or fructose-sweetened beverages (9 weeks) compared with high-complex carbohydrate meals (0 weeks). ${ }^{*} P=0.016$, GLM ANOVA, effect of sugar on $\Delta$ of 16 -hour fractional DNL AUC at 9 weeks vs. 0 weeks. Glucose, $n=8$; fructose, $n=10$. Data represent mean \pm SEM.

fructose-induced postprandial hypertriglyceridemia. (d) Consumption of fructose at $25 \%$ of energy requirements with an ad libitum diet decreased glucose tolerance and insulin sensitivity in older overweight/obese adults compared with glucose consumption. (e) VAT accumulation and increases of 24-hour TG exposure, peak postprandial TG concentrations, and postprandial RLP-C concentrations in response to fructose consumption were more pronounced in men than in women. Consumption of sugar-sweetened beverages resulted in greater decreases in insulin sensitivity in women than in men.

Dose-response studies are needed to determine what levels of dietary fructose and HFCS and/or sucrose are associated with adverse changes of lipids and decreased insulin sensitivity in different populations.

\section{Methods}

Study design. This was a double-blinded parallel arm study that used matched subjects and consisted of 3 phases (Table 1): (a) a 2-week inpatient baseline period during which subjects consumed an energy-balanced diet; (b) an 8 -week outpatient intervention period during which subjects consumed either fructose- or glucose-sweetened beverages providing $25 \%$ of daily energy requirements along with their usual ad libitum diet; and (c) a 2-week inpa- tient intervention period during which subjects consumed fructose- or glucose-sweetened beverages providing $25 \%$ of daily energy requirements with an energy-balanced diet. The inpatient periods allowed comparisons of the high-fructose and glucose diets under well-controlled metabolic conditions. However, sugar-sweetened beverages are typically consumed as part of an ad libitum diet that is likely to contain more energy than the inpatient diet and have the potential to promote weight gain. Therefore, the purpose of the 8-week outpatient period was to compare the effects of consumption of fructose or glucose along with ad libitum diet on BW gain and composition.

Subjects. Participants were recruited through newspaper advertisements and underwent a telephone and an in-person interview with medical history, a complete blood count, and a serum biochemistry panel to assess eligibility. Inclusion criteria included age from 40 to 72 years and BMI of $25-35 \mathrm{~kg} / \mathrm{m}^{2}$ with a self report of stable BW during the prior 6 months. Women were considered postmenopausal based on a self report of no menstruation for at least 1 year. Exclusion criteria included evidence of diabetes, renal disease, or hepatic disease; fasting serum TG concentrations greater than $400 \mathrm{mg} / \mathrm{dl}$; hypertension (>140/90 mmHg); and history of surgery for weight loss. Individuals who smoked, reported exercise of more than 3.5 hours/week at a level more vigorous than walking, or reported having used thyroid, lipid-lowering, glucose-lowering, antihypertensive, antidepressant, or weight-loss medications were also excluded. Diet-related exclusion criteria included habitual ingestion of more than 1 sugar-sweetened beverage per day or more than 2 alcoholic beverages per day. The UCD Institutional Review Board approved the experimental protocol, and subjects provided informed consent for participation in the study. Thirty-nine subjects enrolled in the study, and experimental groups were matched for sexual phenotype, BMI, and fasting TG and insulin concentrations. Seven subjects ( 3 in the glucose group, 4 in the fructose group) did not complete the study because of inability/unwillingness to comply with protocol or due to personal or work-related conflicts.

Diets - inpatient baseline. During the 2-week baseline and 2-week intervention inpatient metabolic phases of the study in the CCRC, subjects consumed energy-balanced diets providing $15 \%$ of energy as protein, $30 \%$ as fat, and 55\% as carbohydrate (Supplemental Table 1). During the baseline period, the carbohydrate content consisted primarily of complex carbohydrates. The diet was designed as a 4-day rotating menu composed of conventional foods served in 3 meals, with $25 \%$ of the energy provided at breakfast (0900 hours), 35\% at lunch (1300 hours), and $40 \%$ at dinner (1800 hours). During the inpatient periods when the diets were controlled and monitored, the subjects were required to consume all of the food and were limited to only the food provided. Daily energy requirements were calculated by the Mifflin equation (67), with an adjustment of 1.3 for activity on the days of the 26-hour isotope infusion and 24-hour serial blood collections and an adjustment of 1.5 for the other inpatient days. BW was monitored daily, and energy intake was adjusted when the slope of the BW

\section{Table 5}

Baseline levels and percentage changes in fasting glucose and insulin and indices of insulin sensitivity after consumption of glucose- or fructose-sweetened beverages for 10 weeks

\section{Outcome variable}

Fasting glucose $(\mathrm{mg} / \mathrm{dl})$

Fasting insulin $(\mu \mathrm{U} / \mathrm{ml})$

Glucose 3-h AUC OGTT $(\mathrm{mg} / \mathrm{dl} \times 3 \mathrm{~h})$

Insulin 3-h AUC OGTT $(\mu \mathrm{U} / \mathrm{ml} \times 3 \mathrm{~h})$

Insulin sensitivity index (mmoles ${ }^{2} \mathrm{H}_{2} \mathrm{O} / 4$-h insulin AUC)

$\begin{array}{cc}\begin{array}{c}\text { Glucose } \\ \text { (0 weeks) }\end{array} & \begin{array}{c}\text { Glucose } \\ \text { (\% change 10 weeks) }\end{array} \\ 87.6 \pm 1.5 & -1.4 \pm 0.6^{\mathrm{A}} \\ 15.0 \pm 1.9 & +2.9 \pm 4.0 \\ 129.4 \pm 16.2 & +31.4 \pm 16.5^{\mathrm{D}} \\ 232.9 \pm 33.0 & +13.9 \pm 9.2 \\ 0.236 \pm 0.036 & +1.1 \pm 8.6\end{array}$

Fructose

(\% change 10 weeks)

$+5.3 \pm 1.0^{\mathrm{B}}$

$+10.2 \pm 4.2^{\mathrm{C}}$

$+60.2 \pm 23.8^{\mathrm{E}}$

$+26.9 \pm 5.9 \mathrm{D}$

$-17.3 \pm 3.8^{\mathrm{E}}$

${ }_{A}^{A} P<0.05 ;{ }^{B} P<0.001 ;{ }^{C} P<0.01$, PROC MIXED 3-factor (time, sexual phenotype, MSRF) RM ANOVA, effect of time. ${ }^{D} P<0.05$; $E P<0.01$, paired Student's $t$ test, 10 weeks vs. 0 weeks. Data represent mean \pm SEM. 
A

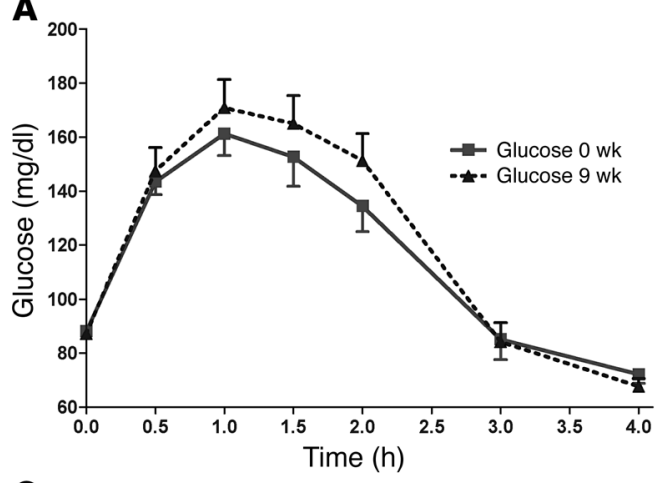

C

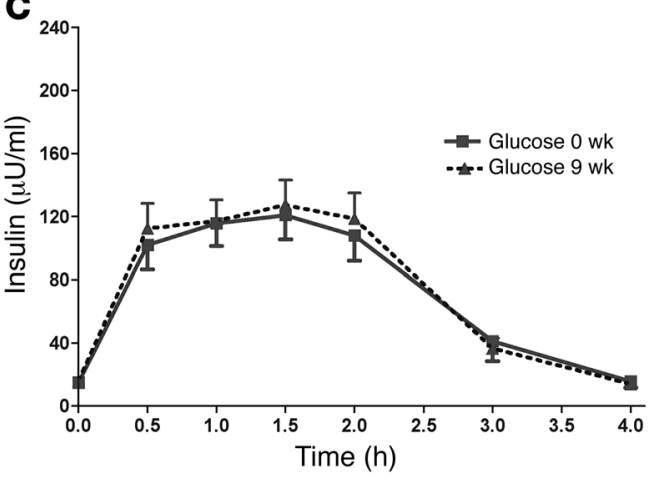

B

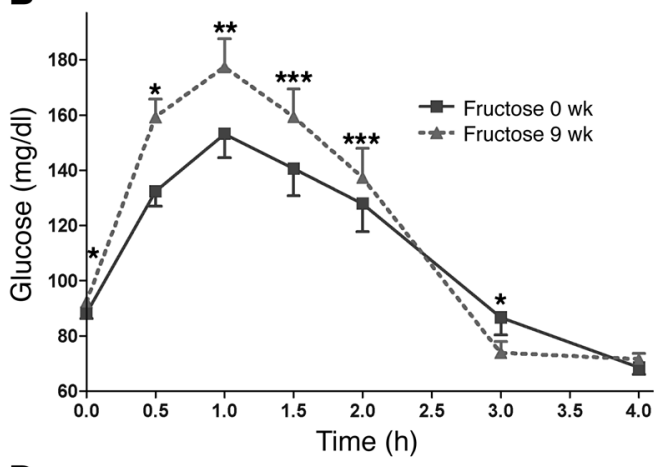

D

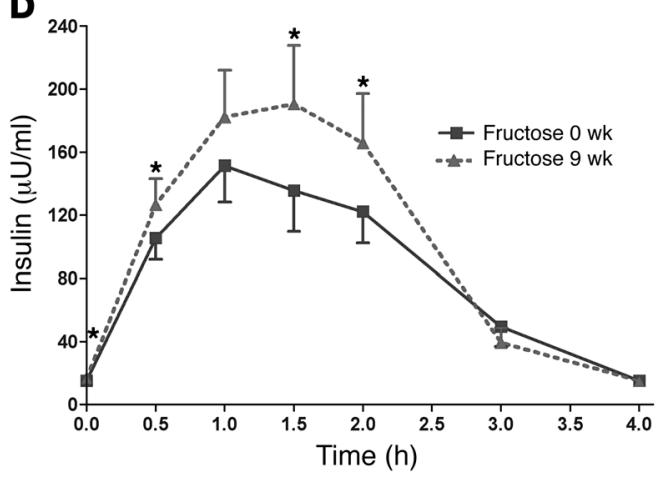

\section{$\mathbf{E}$}

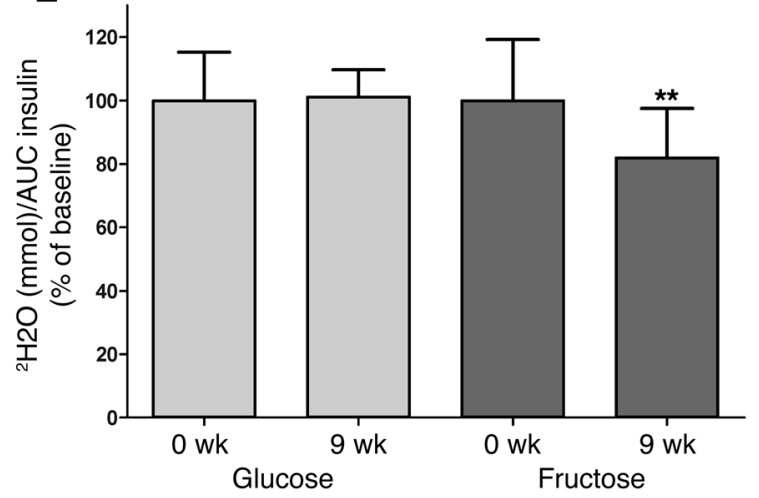

\section{Figure 5}

OGTT and glucose disposal test. Glucose concentrations during an OGTT in subjects before and after 9 weeks of consuming (A) glucose-sweetened beverages or (B) fructose-sweetened beverages. Insulin concentrations during an OGTT in subjects before and after 9 weeks of consuming glucose-sweetened beverages $(\mathbf{C})$ or fructose-sweetened beverages (D). ${ }^{\star} P<0.05 ;{ }^{\star \star} P<0.01$; ${ }^{\star \star \star} P<0.001$, paired Student's $t$ test, 9 weeks vs. 0 weeks. Glucose, $n=15$; fructose, $n=17$. Insulin sensitivity index during glucose disposal test as percentage of baseline in subjects before and after 9 weeks of consuming glucose- or fructose-sweetened beverages $(\mathbf{E})$. ${ }^{\star *} P<0.01$, paired Student's $t$ test, 9 weeks vs. 0 weeks. Glucose: $n=14$; fructose: $n=17$. Data represent mean \pm SEM.

chart trended up or down (68). For 31 of the 32 subjects who completed the study, no adjustments were required.

Diets - outpatient intervention. Subjects were instructed to consume their usual diets. Sugars were provided to the subjects as 3 daily servings of glucose- or fructose-sweetened beverages flavored with an unsweetened drink mix (Kool-Aid; Kraft). Subjects were instructed to drink 3 servings per day, 1 with each meal, and not to consume other sugarcontaining beverages including fruit juice during the study protocol. Beverages were prepared under the supervision of the study supervisor at the UCD Department of Nutrition Ragle Human Nutrition Research Facility. The subjects, CCRC personnel, and technicians who performed analyses were blinded to the sugar assignments. Subjects obtained their beverage supply twice weekly at the CCRC. The beverages contained a biomarker (riboflavin), which was measured fluorometrically in urine samples collected at the time of beverage pickup to monitor compliance. Subjects were informed that they were being monitored for compliance. Based on fluorescein counts, urinary riboflavin levels were $14.6 \pm 1.3$ times higher in subjects consuming glucose and $12.4 \pm 0.8$ times higher in subjects consuming fructose during the intervention weeks than during baseline $(P=0.16)$, which suggests the 2 groups were comparably compliant (Supplemental Table 14).

Estimates of food intake during the outpatient phase of the study were collected by 24-hour recall (via telephone) using the USDA 5-step multiplepass method as described by Conway (69) (Supplemental Table 2). The recalls 


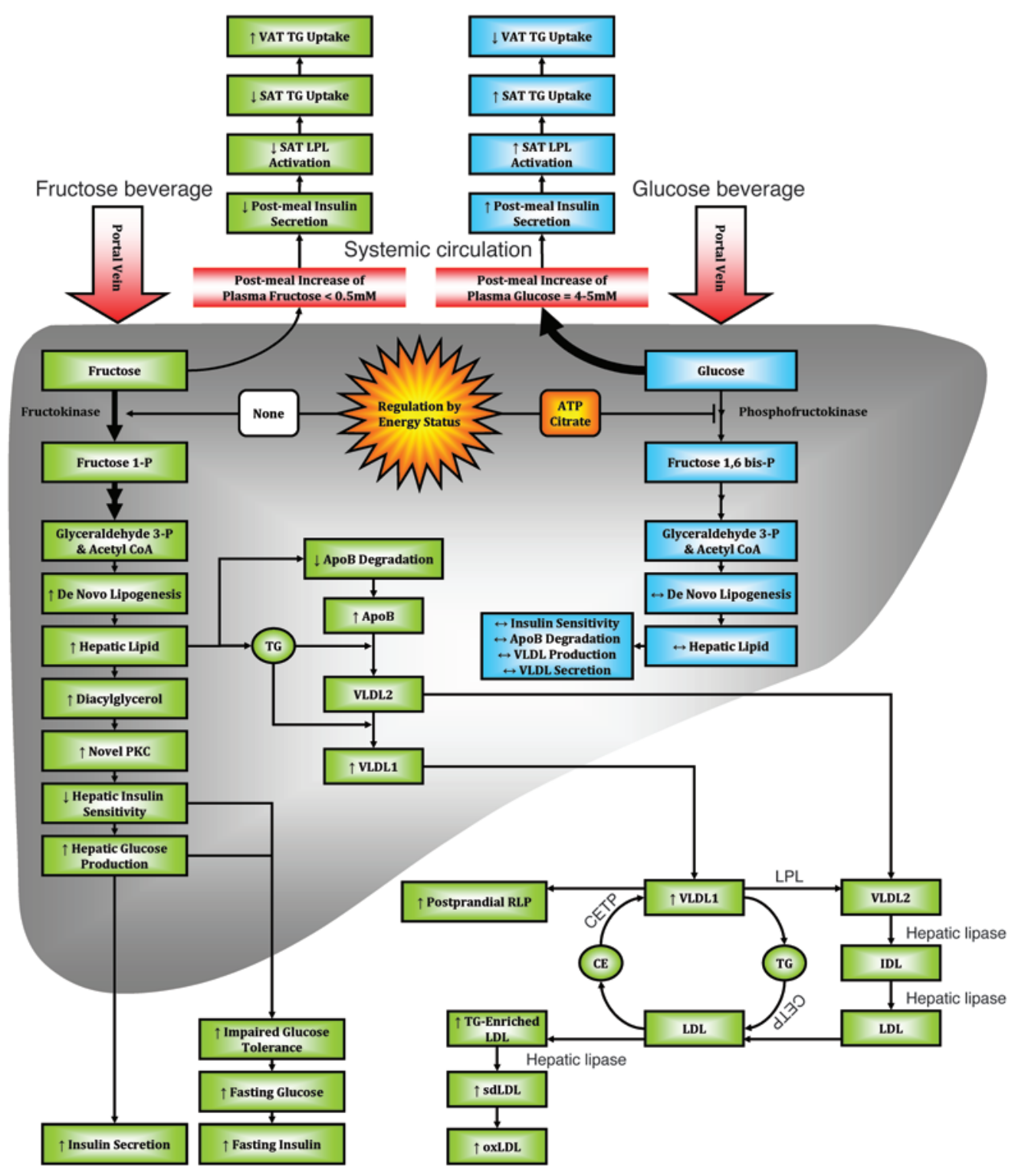

\section{Figure 6}

Proposed mechanisms underlying the differential effects of fructose and glucose consumption. Hepatic glucose metabolism is regulated by phosphofructokinase, which is inhibited by ATP and citrate when energy status is high, thus limiting hepatic uptake of dietary glucose and production of DNL substrates. The hepatic metabolism of dietary fructose is independent of energy status, resulting in unregulated hepatic fructose uptake and increased lipogenesis. The resulting increased hepatic lipid decreases apoB degradation and increases production/secretion of VLDL-TG, mainly as TG-rich VLDL1 (29). This, along with chylomicron competition for LPL-mediated TG hydrolysis and reduced LPL activation by insulin, results in longer VLDL residence time, allowing for augmented cholesteryl ester transfer protein-mediated (CETP-mediated) lipid exchanges with LDL and increased LDL-TG and RLP levels. Hydrolysis of LDL-TG by hepatic lipase increases plasma sdLDL concentrations. After an overnight fast, DNL is no longer elevated and VLDL and chylomicrons remnants have been cleared; thus, plasma TG levels are normal. Postprandially, the increment of plasma apoB levels is associated with VLDL particles; in the fasting state, it is presumably associated with sdLDL, which turns over more slowly. As SAT is more sensitive to insulin activation of LPL activity than VAT, reduced postmeal insulin exposure may lead to less TG uptake in SAT and thus increased TG uptake/accumulation in VAT. Increased hepatic lipid supply may also induce hepatic insulin resistance, possibly through increased levels of diacylglycerol, which activates novel PKC (85). Novel PKC decreases tyrosine phosphorylation of the insulin receptor/insulin receptor substrate 1 , resulting in increased hepatic glucose production, impaired glucose tolerance, and increased fasting glucose and insulin concentrations. oxLDL, oxidized LDL.

were conducted on 6 random days at 2-week and 7-week intervention. The same registered dietitian administered the recall to all subjects. Recalls were analyzed with Nutrition Data System for Research (version 2005, University of Minnesota). The results from all 6 recalls were averaged, except for reports that were judged by the dietitian to be outliers to the usual dietary pattern due to illness or other circumstances.

Diets - inpatient intervention. Following the 8-week outpatient intervention period, subjects returned to the CCRC for a 2-week inpatient inter- 
vention period. The energy-balanced intervention diet was the same as described for the baseline diet, except that while the overall carbohydrate content remained at $55 \%$ of energy requirements, $30 \%$ of the energy was from complex carbohydrates and $25 \%$ was provided by fructose- or glucosesweetened beverages (Supplemental Table 1).

Meals consumed during and prior to the 24 hours in which blood was collected. Meals served during the 24 -hour serial blood collections were identical at all 3 intervention time points ( 2 weeks, 8 weeks, 10 weeks), and the intervention meals were matched as closely as possible to the baseline meals ( 0 weeks), except for the substitution of $25 \%$ of energy from sugars for the complex carbohydrates. The baseline ( 0 weeks) and final (10 weeks) intervention 24-hour serial blood collections were performed after subjects had consumed energy-balanced, weight-maintaining diets in the CCRC for 10 days. The 24-hour serial blood collections that occurred after 2 weeks and 8 weeks of intervention were preceded by 2 - and 8 -week periods of ad libitum food intake. On the day before the 2- and 8-week blood collections, subjects entered the CCRC at 0700 hours and consumed buffet meals ad libitum for breakfast, lunch, and dinner along with the sugar-sweetened beverages. The buffet menu items and quantities provided were the same on both days, and each subject's ad libitum food intake was determined without the subject's being aware that their food intake was monitored.

Measurements of body composition and blood pressure. Subjects were weighed daily in the morning before breakfast during the inpatient phases. Total body fat was determined by dual energy $\mathrm{x}$-ray absorptiometry (DXA). CT scans of the abdomen were performed at the level of the umbilicus to quantify SAT, VAT, and total abdominal fat areas. Total tissue area was computed as the area with an attenuation range of -250 to $+1,500$ Hounsfield units; an attenuation range of -250 to -50 Hounsfield units was used to define fat areas. SAT and VAT areas were differentiated by delineating the border of the peritoneal cavity. CT scans and fat quantifications were performed at the UCD Medical Center under the supervision of John McGahan. Blood pressure was measured with an automatic blood pressure cuff (Welch Allyn) twice daily during inpatient periods.

Gluteal adipose biopsy and RNA analyses. Needle biopsy samples of subcutaneous gluteal adipose tissue were obtained following lidocaine injection. A 3-ml fat biopsy was obtained, transferred to RNAlater (Ambion; Applied Biosystems), and stored at $4^{\circ} \mathrm{C}$. After 48 hours, adipose samples were removed from RNAlater and stored at $-80^{\circ} \mathrm{C}$ until analysis. RNA isolation, cDNA synthesis, and gene expression analysis using TaqMan Gene Expression Assays (Applied Biosystems) were performed as previously described (70) in the laboratory of Ronald Krauss. The percentage changes of gene expression were calculated as the natural log of the expression at 10 weeks/ natural log of the expression at baseline $\times 100$.

24-hour fasting and postprandial blood profiles. 24-hour serial blood collections occurred during baseline ( 0 weeks) and after 2,8 , and 10 weeks of intervention. At 0730 hours, an i.v. catheter was inserted into an arm vein by a registered nurse and kept patent with slow saline infusion. Three fasting blood samples were collected in EDTA at 0800, 0830, and 0900 hours. Thirty-three postprandial blood samples were collected at 30- to 60-minute intervals from 0930 until 0800 hours the next morning $(32,71)$. Meals were served at 0900,1300, and 1800 hours. An additional 3 to $6 \mathrm{ml}$ of blood was collected at each of the following time-points: 0800, 0830, and 0900 and 2200,2300 , and 2330 hours. The plasma from the 3 fasting samples $(0800$, 0830 , and 0900 hours) was pooled, as was the plasma from the 3 postprandial blood samples (2200, 2300, and 2330 hours); multiple aliquots of each pooled sample were stored at $-80^{\circ} \mathrm{C}$.

Hepatic fractional DNL. Fractional DNL was studied via infusion of isotopic acetate during baseline and 10 -week intervention. At 2000 hours, i.v. catheters were inserted into veins of both arms and kept patent with slow saline infusion. Following a baseline blood collection, at 2200 hours, a 26-hour infusion $\left(55.6 \mathrm{ml} / \mathrm{h}\right.$ ) of $0.5 \mathrm{~g} / \mathrm{h}$ sodium $\left[1-{ }^{13} \mathrm{C}\right]$ acetate (Cambridge Isotope Laboratories Inc.) was initiated. Fasting blood samples were collected the next morning at 0700, 0730, and 0800 hours, followed by the initiation of steady-state feeding using a feeding protocol previously validated to study VLDL kinetics (72). Subjects consumed $1 / 16$ of their energy requirement each hour from 0800 to 2300 hours as rice and chicken casseroles with bread during baseline and as rice and chicken casseroles with fructose or glucose beverages during intervention. Postprandial blood was collected hourly from 1300 to 2400 hours. Lipoprotein fractions were isolated using sequential ultracentrifugation (73). Plasma VLDL- ${ }^{13} \mathrm{C}$ palmitate enrichment and mass isotopomers were measured as described by Hellerstein et al. (74) and Faeh et al. (27) in the VLDL1 fraction. Fasting and postprandial hepatic DNL were calculated as the mean of the samples collected before and during the final 10 hours of steady-state feeding, respectively. Due to budget constraints, the stable isotope infusion was conducted only on the first 23 subjects enrolled in the study, and of these tests, 5 were unsuccessful due to nonpatent catheters. There was a disproportionate number of men in the fructose subset ( 7 men, 3 women); however. the effect of sexual phenotype on the general linear model (GLM) 2-factor ANOVA for 16 -hour AUC for fractional DNL was $P=0.89$. Otherwise, there were no significant differences in baseline characteristics between the 2 groups of subjects in the DNL subset, and they were representative of the larger group.

Postheparin LPL. During the baseline and intervention periods, a postprandial blood sample was collected at 2000 hours followed by i.v. injection of 50 units of heparin $/ \mathrm{kg} \mathrm{BW}$ and collection of another blood sample 10 minutes later. The procedure was repeated in the fasting state the following week. Blood samples were assayed for total lipase and hepatic lipase activity by Asahi Kasei Pharma by the method of Imamura (75).

OGTT and disposal test. During baseline and intervention week 9, an OGTT was performed after an overnight fast. Blood samples were collected before and 30, 60, 90, 120, 180, and 240 minutes after consumption of a 75 -gram glucose solution $(300 \mathrm{ml})$. The OGTT was expanded to include measurement of glucose disposal and insulin sensitivity using the deuterated-glucose disposal test developed by Hellerstein and colleagues, as described previously (5). The $75 \mathrm{~g}$ oral glucose load contained $15 \mathrm{~g}$ of $6,6, \mathrm{D}_{2}$ glucose (Cambridge Isotope Laboratories). Because the hydrogen atoms in $\mathrm{C}-\mathrm{H}$ bonds of glucose at position C-6 are more than $90 \%$ lost to tissue water during glycolytic metabolism but are retained in the glucose molecule in the absence of glycolytic metabolism, ${ }^{2} \mathrm{H}_{2} \mathrm{O}$ production provides a sensitive index of whole-body glycolytic utilization of plasma glucose. In addition, glucose transport across capillary endothelium, transport into cells, phosphorylation, and glycolytic metabolism are all increased by insulin (76), and ${ }^{2} \mathrm{H}_{2} \mathrm{O}$ production ( $\mu$ moles)/AUC insulin has been shown to be an insulin-sensitivity index that is highly correlated to the $\mathrm{M}$ value from euglycemic-hyperinsulinemic glucose clamps (5). The deuterium content of plasma samples was determined using a Thermo Finnigan High Temperature Conversion/Elemental Analyzer coupled with a Thermo Finnigan MAT 253 Isotope Ratio-Mass Spectrometer via a ConFlo III Interface. The deuterium isotope abundance was calculated in $\delta^{2} \mathrm{H}$ values relative to the international Vienna Standard Mean Ocean Water standard, transformed to atom percentage excess by using a calibration curve of standards, and converted to millimoles by multiplying the ${ }^{2} \mathrm{H}_{2} \mathrm{O}$ enrichment by the total body water pool size and dividing by 20 (the molecular weight of ${ }^{2} \mathrm{H}_{2} \mathrm{O}$ ). Total body water was measured immediately prior to the OGTT by bioimpedance spectroscopy (Xitron Technologies).

Analyses. Glucose concentrations were measured with an automated glucose analyzer (YSI) and insulin by radioimmunoassay (Millipore). Lipid and lipoprotein concentrations (total cholesterol, HDL, TG, apoB, and apoA1) were determined using a Polychem Chemistry Analyzer (Polymedco Inc.). FFA concentrations were measured with an enzymatic colorimetric assay (Wako) adapted to a microtiter plate. Oxidized LDL was measured with a commercially available ELISA (Mercodia). sdLDL (density = 1.044-1.063 g/ml) 
was separated from plasma by precipitation (77). The LDL concentration of the sdLDL extract and plasma was determined by direct homogenous assay using detergents (LDL-EX; Denka Seiken Co.) (78). The accuracy of this homogenous method has been described $(79,80)$. RLP were quantified with an immunoseparation assay $(81,82)$.

Statistics. The AUC was calculated for TG and DNL using the trapezoidal method. The mean of the 3 baseline values was determined, and the net AUC was calculated by subtracting the AUC values below baseline from the AUC values above baseline. For TG, glucose, and insulin, fasting concentrations represent the mean concentration of the samples collected at 0800, 0830, and 0900 hours during the 24 -hour serial blood collections. For all other lipid parameters, fasting concentrations were measured in pooled plasma samples collected during the 24-hour serial blood collections at 0800, 0830, and 0900 hours, and postprandial levels were measured in pooled plasma collected at 2200, 2300, and 2330 hours. Statistical tests were performed with SAS 9.1.

Differences in the lead-in diet could potentially affect the responses to fructose or glucose consumption at the 3 intervention time points $(2,8$, and 10 weeks) compared with consumption of complex carbohydrates at baseline (0 weeks). Specifically, the 0 - and 10-week 24-hour serial blood collections were preceded by 10 days of consumption of an energy-balanced diet, whereas the 2-week and 8-week collections were preceded by consumption of ad libitum diets. To differentiate the acute variations associated with differences in the prior day's energy intake from the effects of sugar intake, the energy intake of each subject during the 24 hours prior to each 24-hour period in which blood was collected was entered as a timelevel covariable in an RM model using PROC MIXED with time, type of sugar, sexual phenotype, and MSRF as factors. The covariable was removed from the model when its $F$ statistic was $\leq 1$.0. Insignificant 3-way interactions were removed if they decreased the precision of the model. MSRF was defined by the American Heart Association/National Heart, Lung, and Blood Institute $(83,84)$. Response variables were further analyzed using the same model in separate RM ANOVAs for fructose or glucose with time, sexual phenotype, and MSRF as factors. For both the 3- and 4-factor RM ANOVAs, Tukey's multiple comparison post tests were used to compare effects of type of sugar, sexual phenotype, or MSRF within and between groups. For response variables that were measured at only 2 time points, 0 and 10 weeks, the $\Delta$ or percentage $\Delta$ between the 2 time points was analyzed by GLM, with type of sugar, sexual phenotype, and MSRF as factors. The effects of sexual phenotype and MSRF within the individual sugar groups were analyzed in sugar-specific 2-factor GLM ANOVAs with Tukey's multiple comparison tests, and the effects of the individual sugars were analyzed by 2 -tailed paired Student's $t$ test ( 10 vs. 0 weeks). $P<0.05$ was considered significant. Data are presented as mean \pm SEM.

Note added in proof. A new manuscript from our group appeared recently (86) that extends our previous study from 2004 (32) of the effects of shortterm ( 1 day) fructose and glucose consumption in normal weight young women to young, obese men and women and examines the effects of baseline insulin sensitivity (HOMA-IR) on the acute 24 -hour TG response. The current study provides data on the effects of sustained long-term fructose and glucose exposure on dyslipidemia, de novo lipogenesis, insulin sensitivity, and visceral adiposity.

\section{Acknowledgments}

The authors thank Marinelle Nuñez, Theresa Tonjes, Brandi Bair, Rebecca Stewart, Sara Wuehler, Elaine Souza, and Patrick Lam for their excellent technical support and Nicole Mullen and the nursing staff at CCRC for their dedicated nursing support. We thank Shigeyuki Imamura for analyses of postheparin LPL activity. We also thank Jorge Dubcovsky and Iago Lowe for guidance and Janet Peerson for expert advice on the statistical analysis of the data. This research was supported with funding from NIH grant R01 HL-075675. The project also received support from grant number UL1 RR024146 from the National Center for Research Resources (NCRR), a component of the NIH, and the NIH Roadmap for Medical Research. P.J. Havel's laboratory also receives support from NIH grants HL-091333, AT-002599, AT-002993, and AT-003545 and the American Diabetes Association. N.L. Keim's research is supported by intramural USDA-ARS CRIS 5306-51530-016-00D. We are grateful to Janet King of Children's Hospital Oakland Research Institute for her ongoing support of this research.

Received for publication September 8, 2008, and accepted in revised form February 11, 2009.

Address correspondence to: Peter J. Havel, Department of Molecular Biosciences, School of Veterinary Medicine, University of California, Davis, One Shields Avenue, Davis, California 95616, USA. Phone: (530) 752-6553; Fax: (530) 752-2474; E-mail: pjhavel@ucdavis.edu.
1. Bizeau, M.E., and Pagliassotti, M.J. 2005. Hepatic adaptations to sucrose and fructose. Metabolism. 54:1189-1201.

2. Havel, P.J. 2005. Dietary fructose: implications for dysregulation of energy homeostasis and lipid/carbohydrate metabolism. Nutr. Rev. 63:133-157.

3. Le, K.A., and Tappy, L. 2006. Metabolic effects of fructose. Curr. Opin. Clin. Nutr. Metab. Care. 9:469-475.

4. Wei, Y., Wang, D., Topczewski, F., and Pagliassotti, M.J. 2007. Fructose-mediated stress signaling in the liver: implications for hepatic insulin resistance. J. Nutr. Biochem. 18:1-9.

5. Beysen, C., et al. 2007. Whole-body glycolysis measured by the deuterated-glucose disposal test correlates highly with insulin resistance in vivo. Diabetes Care. 30:1143-1149.

6. Bantle, J.P., Swanson, J.E., Thomas, W., and Laine, D.C. 1992. Metabolic effects of dietary fructose in diabetic subjects. Diabetes Care. 15:1468-1476.

7. Crapo, P.A., and Kolterman, O.G. 1984. The metabolic effects of 2-week fructose feeding in normal subjects. Am. J. Clin. Nutr. 39:525-534.

8. Koivisto, V.A., and Yki-Jarvinen, H. 1993. Fructose and insulin sensitivity in patients with type 2 diabetes. J. Intern. Med. 233:145-153.

9. Reiser, S., Bickard, M.C., Hallfrisch, J., Michaelis,
O.E., 4th, and Prather, E.S. 1981. Blood lipids and their distribution in lipoproteins in hyperinsulinemic subjects fed three different levels of sucrose. J. Nutr. 111:1045-1057.

10. Swanson, J.E., Laine, D.C., Thomas, W., and Bantle, J.P. 1992. Metabolic effects of dietary fructose in healthy subjects. Am. J. Clin. Nutr. 55:851-856.

11. Swarbrick, M.M., et al. 2008. Consumption of fructose-sweetened beverages for 10 weeks increases postprandial triacylglycerol and apolipoprotein-B concentrations in overweight and obese women. Br. J. Nutr. 100:947-952.

12. Turner, J.L., Bierman, E.L., Brunzell, J.D., and Chait, A. 1979. Effect of dietary fructose on triglyceride transport and glucoregulatory hormones in hypertriglyceridemic men. Am. J. Clin. Nutr. 32:1043-1050.

13. Bantle, J.P., Raatz, S.K., Thomas, W., and Georgopoulos, A. 2000. Effects of dietary fructose on plasma lipids in healthy subjects. Am. J. Clin. Nutr. 72:1128-1134.

14. Crapo, P.A., Kolterman, O.G., and Henry, R.R. 1986. Metabolic consequence of two-week fructose feeding in diabetic subjects. Diabetes Care. 9:111-119.

15. Hallfrisch, J., et al. 1983. Effects of dietary fructose on plasma glucose and hormone responses in normal and hyperinsulinemic men. J. Nutr. 113:1819-1826.
16. Herman, R.H., Zakim, D., and Stifel, F.B. 1970. Effect of diet on lipid metabolism in experimental animals and man. Fed. Proc. 29:1302-1307.

17. Le, K.A., et al. 2006. A 4-wk high-fructose diet alters lipid metabolism without affecting insulin sensitivity or ectopic lipids in healthy humans. Am. J. Clin. Nutr. 84:1374-1379.

18. Reiser, S., et al. 1989. Day-long glucose, insulin, and fructose responses of hyperinsulinemic and nonhyperinsulinemic men adapted to diets containing either fructose or high-amylose cornstarch. Am.J. Clin. Nutr. 50:1008-1014.

19. Stanhope, K.L., et al. 2008. Twenty-four-hour endocrine and metabolic profiles following consumption of high-fructose corn syrup-, sucrose-, fructose-, and glucose-sweetened beverages with meals. Am. J. Clin. Nutr. 87:1194-1203.

20. Nabeno, Y., Fukuchi, Y., Matsutani, Y., and Naito, M. 2007. Influence of aging and menopause on postprandial lipoprotein responses in healthy adult women. J. Atheroscler. Thromb. 14:142-150.

21. Kolovou, G.D., et al. 2004. The influence of natural menopause on postprandial lipemia in heterozygotes for familial hypercholesterolemia. J. Womens Health. (Larchmt.) 13:1119-1126.

22. Kolovou, G.D., and Bilianou, H.G. 2008. Influence 
of aging and menopause on lipids and lipoproteins in women. Angiology. 59:54S-57S

23. Mayes, P.A. 1993. Intermediary metabolism of fructose Am. J. Clin. Nutr. 58:754S-765S.

24. Matsuzaka, T., et al. 2004. Insulin-independent induction of sterol regulatory element-binding protein-1c expression in the livers of streptozotocin-treated mice. Diabetes. 53:560-569.

25. Nagai, Y., et al. 2002. Amelioration of high fructose-induced metabolic derangements by activation of PPARalpha. Am. J. Physiol. Endocrinol. Metab. 282:E1180-E1190

26. Schwarz,J.M., Neese, R.A., Schakleton, C., and Hellerstein, M.K. 1993. De novo lipogenesis during fasting and oral fructose ingestion in lean and obese hyperinsulinemic subjects [abstract]. Diabetes. 42:A39.

27. Faeh, D., et al. 2005. Effect of fructose overfeeding and fish oil administration on hepatic de novo lipogenesis and insulin sensitivity in healthy men. Diabetes. 54:1907-1913.

28. McGarry, J.D. 1995. Malonyl-CoA and carnitine palmitoyltransferase I: an expanding partnership. Biochem. Soc. Trans. 23:481-485.

29. Adiels, M., et al. 2006. Overproduction of large VLDL particles is driven by increased liver fat content in man. Diabetologia. 49:755-765.

30. Olofsson, S.O., and Boren, J. 2005. Apolipoprotein B: a clinically important apolipoprotein which assembles atherogenic lipoproteins and promotes the development of atherosclerosis. J. Intern. Med. 258:395-410.

31. Adiels, M., Olofsson, S.O., Taskinen, M.R., and Boren, J. 2008. Overproduction of very low-density lipoproteins is the hallmark of the dyslipidemia in the metabolic syndrome. Arterioscler. Thromb. Vasc. Biol. 28:1225-1236.

32. Teff, K.L., et al. 2004. Dietary fructose reduces circulating insulin and leptin, attenuates postprandial suppression of ghrelin, and increases triglycerides in women. J. Clin. Endocrinol. Metab. 89:2963-2972.

33. Mead, J.R., Irvine, S.A., and Ramji, D.P. 2002. Lipoprotein lipase: structure, function, regulation, and role in disease. J. Mol. Med. 80:753-769.

34. Fried, S.K., Russell, C.D., Grauso, N.L., and Brolin, R.E. 1993. Lipoprotein lipase regulation by insulin and glucocorticoid in subcutaneous and omental adipose tissues of obese women and men. J. Clin. Invest. 92:2191-2198

35. Bansal, S., et al. 2007. Fasting compared with nonfasting triglycerides and risk of cardiovascular events in women. JAMA. 298:309-316.

36. Hyson, D., Rutledge, J.C., and Berglund, L. 2003. Postprandial lipemia and cardiovascular disease. Curr. Atheroscler. Rep. 5:437-444.

37. Karpe, F. 1999. Postprandial lipoprotein metabolism and atherosclerosis. J. Intern. Med. 246:341-355.

38. Lopez-Miranda, J., et al. 2006. Postprandial lipoprotein metabolism, genes and risk of cardiovascular disease. Curr. Opin. Lipidol. 17:132-138.

39. Nordestgaard, B.G., Benn, M., Schnohr, P., and Tybjaerg-Hansen, A. 2007. Nonfasting triglycerides and risk of myocardial infarction, ischemic heart disease, and death in men and women. JAMA. 298:299-308.

40. Stalenhoef, A.F., and de Graaf, J. 2008. Association of fasting and nonfasting serum triglycerides with cardiovascular disease and the role of remnantlike lipoproteins and small dense LDL. Curr. Opin. Lipidol. 19:355-361.

41. Berneis, K.K., and Krauss, R.M. 2002. Metabolic origins and clinical significance of LDL heterogeneity. J. Lipid Res. 43:1363-1379.

42. Krauss, R.M. 2005. Dietary and genetic probes of atherogenic dyslipidemia. Arterioscler. Thromb. Vasc. Biol. 25:2265-2272.

43. Marcovina, S., and Packard, C.J. 2006. Measurement and meaning of apolipoprotein $\mathrm{AI}$ and apolipoprotein B plasma levels. J. Intern. Med. 259:437-446.

44. Packard, C.J. 2003. Triacylglycerol-rich lipoproteins and the generation of small, dense low-density lipo- protein. Biochem. Soc. Trans. 31:1066-1069.

45. Packard, C.J. 2006. Small dense low-density lipoprotein and its role as an independent predictor of cardiovascular disease. Curr. Opin. Lipidol. 17:412-417.

46. Rutledge, A.C., and Adeli, K. 2007. Fructose and the metabolic syndrome: pathophysiology and molecular mechanisms. Nutr. Rev. 65:S13-S23.

47. Bergman, R.N., et al. 2007. Abdominal obesity: role in the pathophysiology of metabolic disease and cardiovascular risk. Am. J. Med. 120:S3-S8; discussion S29-S32.

48. Stanhope, K.L., and Havel, P.J. 2008. Fructose consumption: potential mechanisms for its effects to increase visceral adiposity and induce dyslipidemia and insulin resistance. Curr. Opin. Lipidol. 19:16-24.

49. Morino, K., Petersen, K.F., and Shulman, G.I. 2006. Molecular mechanisms of insulin resistance in humans and their potential links with mitochondrial dysfunction. Diabetes. 55(Suppl. 2):S9-S15.

50. Seppala-Lindroos, A., et al. 2002. Fat accumulation in the liver is associated with defects in insulin suppression of glucose production and serum free fatty acids independent of obesity in normal men. J. Clin. Endocrinol. Metab. 87:3023-3028.

51. Newton, A.C. 2003. Regulation of the ABC kinases by phosphorylation: protein kinase $\mathrm{C}$ as a paradigm. Biochem. J. 370:361-371.

52. Itani, S.I., Ruderman, N.B., Schmieder, F., and Boden, G. 2002. Lipid-induced insulin resistance in human muscle is associated with changes in diacylglycerol, protein kinase C, and IkappaB-alpha. Diabetes. 51:2005-2011.

53. Yu, C., et al. 2002. Mechanism by which fatty acids inhibit insulin activation of insulin receptor substrate-1 (IRS-1)-associated phosphatidylinositol 3-kinase activity in muscle. J. Biol. Chem. 277:50230-50236

54. Beck-Nielsen, H., Pedersen, O., and Lindskov, H.O. 1980. Impaired cellular insulin binding and insulin sensitivity induced by high-fructose feeding in normal subjects. Am. J. Clin. Nutr. 33:273-278.

55. Couchepin, C., et al. 2008. Markedly blunted metabolic effects of fructose in healthy young female subjects compared with male subjects. Diabetes Care. 31:1254-1256.

56. Gibbons, G.F., Islam, K., and Pease, R.J. 2000. Mobilisation of triacylglycerol stores. Biochim. Biophys. Acta. 1483:37-57.

57. Toth, M.J., and Tchernof, A. 2000. Lipid metabolism in the elderly. Eur. J. Clin. Nutr. 54(Suppl. 3):S121-S125.

58. Guthrie, J.F., and Morton, J.F. 2000. Food sources of added sweeteners in the diets of Americans. J. Am. Diet Assoc. 100:43-51.

59. Mundt, C.A., et al. 2006. Relationships of activity and sugar drink intake on fat mass development in youths. Med. Sci. Sports Exerc. 38:1245-1254.

60. Storey, M.L., Forshee, R.A., and Anderson, P.A 2006. Beverage consumption in the US population. J. Am. Diet Assoc. 106:1992-2000.

61. Striegel-Moore, R.H., et al. 2006. Correlates of beverage intake in adolescent girls: the National Heart, Lung, and Blood Institute Growth and Health Study. J. Pediatr. 148:183-187.

62. van der Horst, K., et al. 2007. Perceived parenting style and practices and the consumption of sugarsweetened beverages by adolescents. Health Educ. Res. 22:295-304.

63. West, D.S., et al. 2006. Self-reported sugar-sweetened beverage intake among college students. Obesity (Silver Spring). 14:1825-1831.

64. Matsuura, E., Hughes, G.R., and Khamashta, M.A. 2008. Oxidation of LDL and its clinical implication. Autoimmun. Rev. 7:558-566.

65. Nakajima, K., Nakano, T., and Tanaka, A. 2006. The oxidative modification hypothesis of atherosclerosis: the comparison of atherogenic effects on oxidized LDL and remnant lipoproteins in plasma.
Clin. Chim. Acta. 367:36-47.

66. Walldius, G., and Jungner, I. 2006. The apoB/apoA-I ratio: a strong, new risk factor for cardiovascular disease and a target for lipid-lowering therapy--a review of the evidence. J. Intern. Med. 259:493-519.

67. Mifflin, M.D., et al. 1990. A new predictive equation for resting energy expenditure in healthy individuals. Am. J. Clin. Nutr. 51:241-247.

68. Keim, N.L., Stern, J.S., and Havel, P.J. 1998. Relation between circulating leptin concentrations and appetite during a prolonged, moderate energy deficit in women. Am. J. Clin. Nutr. 68:794-801.

69. Conway, J.M., Ingwersen, L.A., Vinyard, B.T., and Moshfegh, A.J. 2003. Effectiveness of the US Department of Agriculture 5-step multiple-pass method in assessing food intake in obese and nonobese women. Am. J. Clin. Nutr. 77:1171-1178.

70. Mangravite, L.M., Dawson, K., Davis, R.R., Gregg, J.P., and Krauss, R.M. 2007. Fatty acid desaturase regulation in adipose tissue by dietary composition is independent of weight loss and is correlated with the plasma triacylglycerol response. Am. J. Clin. Nutr. 86:759-767.

71. Havel, P.J., Townsend, R., Chaump, L., and Teff, K. 1999. High-fat meals reduce 24-h circulating leptin concentrations in women. Diabetes. 48:334-341.

72. Lichtenstein, A.H., et al. 1990. Comparison of deuterated leucine, valine, and lysine in the measurement of human apolipoprotein A-I and B-100 kinetics. J. Lipid Res. 31:1693-1701.

73. Karpe, F., Steiner, G., Uffelman, K., Olivecrona, T., and Hamsten, A. 1994. Postprandial lipoproteins and progression of coronary atherosclerosis. Atherosclerosis. 106:83-97.

74. Hellerstein, M.K., et al. 1991. Measurement of de novo hepatic lipogenesis in humans using stable isotopes. J. Clin. Invest. 87:1841-1852.

75. Imamura, S., et al. 2007. A novel method for measuring human hepatic lipase activity in postheparin plasma. J. Lipid Res. 48:453-457.

76. Williams, K.V., et al. 2003. Glucose transport and phosphorylation in skeletal muscle in obesity: insight from a muscle-specific positron emission tomography model. J. Clin. Endocrinol. Metab. 88:1271-1279.

77. Hirano, T., Ito, Y., and Yoshino, G. 2005. Measurement of small dense low-density lipoprotein particles. J. Atheroscler. Thromb. 12:67-72

78. Hirano, T., Ito, Y., Saegusa, H., and Yoshino, G. 2003. A novel and simple method for quantification of small, dense LDL. J. Lipid Res. 44:2193-2201.

79. Okada, M., Matsui, H., Ito, Y., Fujiwara, A., and Inano, K. 1998. Low-density lipoprotein cholesterol can be chemically measured: a new superior method. J. Lab. Clin. Med. 132:195-201.

80. Sakaue, T., et al. 2000. Reactions of direct LDLcholesterol assays with pure LDL fraction and IDL: comparison of three homogeneous methods. Clin. Chim. Acta. 295:97-106.

81. Marcoux, C., et al. 1998. Plasma remnant-like particle lipid and apolipoprotein levels in normolipidemic and hyperlipidemic subjects. Atherosclerosis. 139:161-171.

82. Nakajima, K., et al. 1994. A new approach for the detection of type III hyperlipoproteinemia by RLPcholesterol assay. J. Atheroscler. Thromb. 1:30-36.

83. Al-Daghri, N.M., Al-Attas, O.S., Al-Rubeaan, K., and Sallam, R. 2008. Adipocytokine profile of type 2 diabetics in metabolic syndrome as defined by various criteria. Diabetes Metab. Res. Rev. 24:52-58.

84. Genuth, S., et al. 2003. Follow-up report on the diagnosis of diabetes mellitus. Diabetes Care. 26:3160-3167.

85. Samuel, V.T., et al. 2007. Inhibition of protein kinase Cepsilon prevents hepatic insulin resistance in nonalcoholic fatty liver disease. J. Clin. Invest. 117:739-745.

86. Teff, K.L., et al. 2009. Endocrine and metabolic effects of consuming fructose- and glucose-sweetened beverages with meals in obese men and women: influence of insulin resistance on plasma triglyceride responses. J. Clin. Endocrinol. Metab. doi:10.1210/jc.2008-2192. 